ESAIM: COCV 27 (2021) 77

https://doi.org/10.1051/cocv/2021075
ESAIM: Control, Optimisation and Calculus of Variations

www.esaim-cocv.org

\title{
A MASS REDUCING FLOW FOR REAL-VALUED FLAT CHAINS WITH APPLICATIONS TO TRANSPORT NETWORKS*
}

\author{
Carol Ann Downes**
}

\begin{abstract}
An oriented transportation network can be modeled by a 1-dimensional chain whose boundary is the difference between the demand and supply distributions, represented by weighted sums of point masses. To accommodate efficiencies of scale into the model, one uses a suitable $\mathbf{M}^{\alpha}$ norm for transportation cost for $\alpha \in(0,1]$. One then finds that the minimal cost network has a branching structure since the norm favors higher multiplicity edges, representing shared transport. In this paper, we construct a continuous flow that evolves some initial such network to reduce transport cost without altering its supply and demand distributions. Instead of limiting our scope to transport networks, we construct this $\mathbf{M}^{\alpha}$ mass reducing flow for real-valued flat chains by finding a higher dimensional real chain whose slices dictate the flow. Keeping the boundary fixed, this flow reduces the $\mathbf{M}^{\alpha}$ mass of the initial chain and is Lipschitz continuous under the flat- $\alpha$ norm. To complete the paper, we apply this flow to transportation networks, showing that the flow indeed evolves branching transport networks to be more cost efficient.
\end{abstract}

Mathematics Subject Classification. 49Q20, 49Q10.

Received May 26, 2020. Accepted June 28, 2021.

\section{INTRODUCTION}

Transport theory as in the Monge-Kantorovich problem $(1781 ; 1942)$ focuses on minimizing transportation cost in a way that depends only on optimally allocating or distributing goods $[26,28]$. Here, the cost of a transport plan is typically an integral of some convex function of distance, such as $|x-y|^{p}$ for $1 \leq p<\infty$. And so, the functional does not account for the actual path traveled by the goods; one can assume each good is transported along a straight line connecting its source to its destination.

While this model allows for easier analysis, it is not realistic for many practical problems. As noted by Xia [41], the cost of transportation in real applications is rarely completely determined by the distance between the supply and demand locations. Instead, the physical transport path influences total cost because "carpooling" encourages overlapping in cost efficient ways, making ramified structures preferable. This is due to the fact that in operations there are often efficiencies of scale-i.e., the cost per pound for transporting freight decreases as the amount of freight increases.

*I wish to express my gratitude to Rice University for their support during much of this research, and specifically, Dr. Robert Hardt for his guidance. I'd also like to thank Dr. Christopher Camfield for his thoughts during the writing of this paper. Finally, I thank the referees for their insights and corrections.

Keywords and phrases: Optimal transport networks, mass reducing flows, flat chains.

Hendrix College, 1600 Washington Avenue, Conway, AR 72032-3080, USA.

** Corresponding author: downes@hendrix.edu 
The first to accommodate efficiencies of scale into models of transportation cost was Gilbert, who represented the amount of mass traveling along the edge $e$ by its capacity $\lambda(e)$ [22]. Then, he required the cost per unit length of an edge with capacity $\lambda(e)$, denoted $f(\lambda)$, to be subadditive and increasing. That is, $f\left(\lambda_{1}\right)+f\left(\lambda_{2}\right) \geq$ $f\left(\lambda_{1}+\lambda_{2}\right) \geq \max \left\{f\left(\lambda_{1}\right), f\left(\lambda_{2}\right)\right\}$. The simplest such cost is $f(\lambda)=\lambda^{\alpha}$ for $\alpha \in[0,1]$. Modeling branched transport networks is extensively explored in $[5,27,35,41-50]$.

However, finding the optimal branch structure proves difficult, even in the case with finite supply and demand distributions. The difficulty lies in locating the bifurcation points that optimally take advantage of the cost benefits afforded by $\alpha$. In his $\mathrm{PhD}$ thesis, Bernot gave a construction for an optimal transport path within a fixed, full topology [6]. To find a global minimum, one would need to apply this construction to every possible topology. Not only does this process become computationally unfeasible for a large number of sources/sinks, it is often unclear how to apply the process when the topology has degeneracies.

Others have explored how to approximate optimal shapes of branched transport networks. Oudet and Santambrogio employed a Modica-Mortola approximation method adapted for branched transport in [30]. Pegon, Santambrogio, and Xia in [31] numerically approximated minimal paths from one source via an adaption of phase-field approximation techniques.

Instead of solving this optimization problem outright or following the numerical approximation techniques above, we create a cost reducing flow to evolve transport networks. The underlying theme of geometric flows is to gain insight into such difficult optimization problems instead of attempting to solve the problem directly. For example, the mean curvature flow minimizes the area functional by evolving hypersurfaces via the gradient flow for surface area [10,21, 23, 25, 39]. Brakke extended mean curvature flow to varifolds [7], and similarly, Evans and Spruck evolved level sets of viscosity solutions of suitable differential equations [13-16].

Others have used a time discretization process to create minimizing flows. For example, Cheng developed a mass reducing flow for integral currents [8]. Almgren and Taylor created a flat flow defined by a variety of integrands including crystalline in [3] and with Wang in [4]. Similarly, in 2004, Haga, Hoshino, and Kikuchi constructed a harmonic map flow [24]. Our flow also follows a time discretization process to help solve the motivating problem of evolving transport networks.

Instead of restricting our view to transport networks, we conveniently define the flow for real-valued, $k$ dimensional flat chains, which are domains of integration generalizing oriented manifolds, as conceptualized by Whitney in 1957 [36]. Besides reducing the $\mathbf{M}^{\alpha}$ mass of chains, the resulting flow is Lipschitz continuous in time with respect to a flat- $\alpha$ norm.

The idea of the proof is to generalize Cheng's work for integral currents [8]. First, we construct discrete sequences that minimize a functional related to a scaled flat- $\alpha$ norm. Then, via Brian White's compactness theorem [38], we find a $(k+1)$-dimensional, real-valued chain, whose slices dictate the flow.

After constructing the flow, we explore applications to transportation networks. We flow an initial transport path to a local minimum by viewing transportation networks as 1-dimensional, real-valued flat chains and their transport costs as their $\mathbf{M}^{\alpha}$ masses, as in Bernot, Caselles, and Morel's book [5] and in the work of Xia [41-50]. In fact, the minimizing flow encourages overlapping structures, naturally changing the topology of the initial network through a "(un)zipping" process which creates or eliminates interior vertices. (See the motivating example in Fig. 1.) Because of this and the fact that the flow fixes a chain's boundary, it is particularly amenable to help find optimal transport networks.

\section{Preliminaries}

Flat chains generalize the concept of oriented manifolds, possibly with boundary. Their definition originates from investigating the following problem called the Plateau problem: given a closed, $(k-1)$-dimensional boundary $\Gamma \subset \mathbb{R}^{m}$, find a $k$-dimensional surface $S$ of least $k$-dimensional area among all surfaces with $\partial S=\Gamma$.

This problem was inspired by the Belgian physicist Joseph Plateau's (1801-1883) experiments with soap films [32]. In fact, questions about existence and regularity for a $k$-dimensional Plateau solution became the key motivation for the modern development of the field of geometric measure theory. 


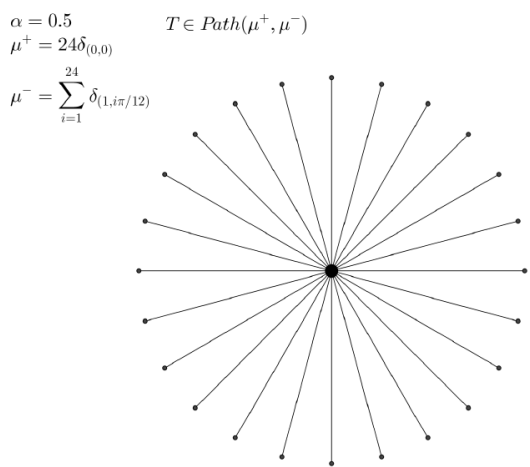

$\mathbf{M}^{\alpha}(T)=24$

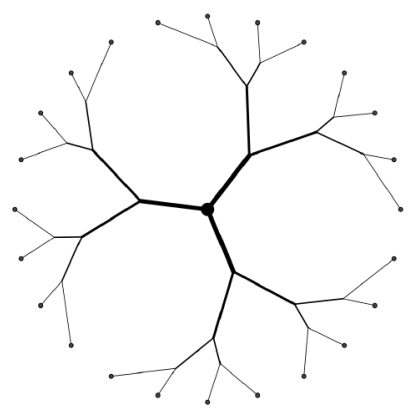

$\mathbf{M}^{\alpha}(T)=16.41$

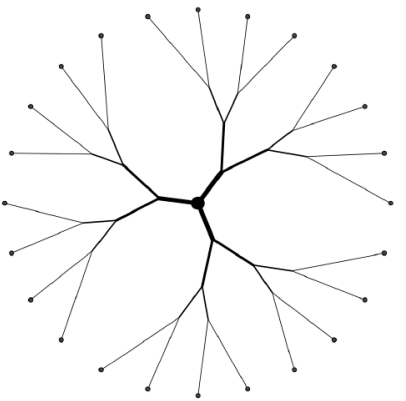

$\mathbf{M}^{\alpha}(T)=18.19$

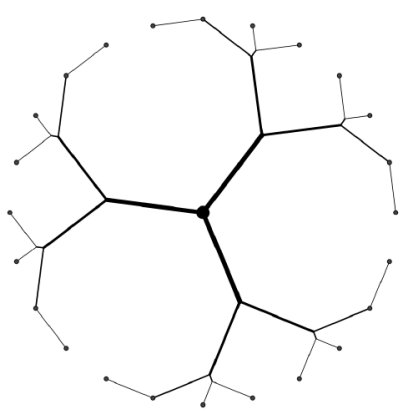

$\mathbf{M}^{\alpha}(T)=15.93$

FiguRE 1. Here are snapshots of the flow on a transport path from one central Dirac mass (of weight twenty-four) to twenty-four equally spaced unit Dirac masses; here $\alpha=0.5$.

To show the existence of a minimal surface $S$ with the desired boundary, one might initially try to implement the direct method of calculus of variations - taking a minimizing sequence of surfaces with boundary $\Gamma$ and extracting a subsequence converging to the solution surface. This would require existence of finite area surfaces with boundary $\Gamma$, lower semicontinuity of area with respect to some topology, and compactness with respect to this topology. Additionally, one needs continuity of the boundary operator to ensure that the boundary stays fixed. However, Figure 2 demonstrates how compactness of surfaces in $\mathbb{R}^{3}$ can go awry.

Hence, mathematicians had to develop new strategies to answer the Plateau problem. In the 1930's, Douglas and Rado independently solved the problem for 2-dimensional surfaces that can be parameterized by a single disk; in particular, they showed that every smooth Jordan curve bounds a mapped-in disc of least mapping area $[11,33]$. Since their work heavily relies on conformal parameterizations of surfaces, it limits the types of possible singularities in solutions. In 1960, Reifenberg solved a formulation of the Plateau problem, but subject to certain topological constraints [34]. A natural objection to these solutions is that one must eliminate conceivable solutions that form in actual soap film experiments because of a priori imposed assumptions on achievable singularities and topological complexities.

To circumvent many of these restrictions, Federer and Fleming offered a solution to the Plateau problem for arbitrary dimension and codimension by using their newly defined normal and integral currents [18]. Fleming gave another approach in 1966 using different generalized oriented manifolds, called flat chains [20]. While avoiding the a priori assumptions on singularities and topological complexities, both currents and flat chains 


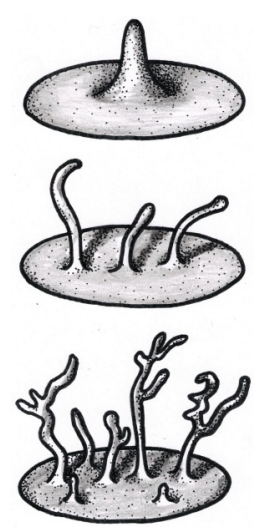

Figure 2. The space of surfaces (e.g. mappings) may fail to be compact with respect to a considered topology. Depicted above are three surfaces from a sequence with the unit circle as boundary whose surface areas decrease towards $\pi$. However, since each surface gains more and more thin tentacles, the limit could include all of $\mathbb{R}^{3}$. See [29].

offer natural topologies with compactness properties, and thus, surmount the issue in implementing the direct method of calculus of variations.

For more information on the history of the Plateau problem and its contribution to the founding of geometric measure theory, see $[2,29]$.

One may consider finding an optimal transportation network as solving an $\mathbf{M}^{\alpha}$ mass generalization of the 1dimensional Plateau problem. Motivated by this, we create an $\mathbf{M}^{\alpha}$ mass reducing flow on real-valued flat chains. But first we define in this section flat chains and develop the preliminary material necessary for creating the flow. Sources that provide much of the background presented here include [12, 17, 20, 29, 36-38]. In particular, we follow the approach of Fleming [20] and White [37, 38, 40] in which we define flat chains for real coefficients with norm $|g|^{\alpha}$ for $\alpha \in[0,1]$ as the completion with respect to a flat metric of polyhedral chains. Chodosh's lecture notes from Brian White's Topics in Geometric Measure Theory course from Spring 2012 [9] provide an excellent expository treatment of the topics below including an equivalent definition to our fill- $\alpha$ norm defined in this section.

\subsection{Flat chains, $M^{\alpha}$ mass, and the flat- $\alpha$ seminorm}

Let $K \subset \mathbb{R}^{m}$ be a compact set in $m$-dimensional Euclidean space and let $k \in\{0,1, \ldots, m\}$.

Definition 2.1. Let $\mathcal{P}_{k}(K)$ denote the group of polyhedral chains of dimension $k$ with real coefficients and support in $K$. We may write any polyhedral chain in $\mathcal{P}_{k}(K)$ as $P=\sum g_{i} \sigma_{i}$ where each $g_{i} \in \mathbb{R}$ and $\left\{\sigma_{i}\right\}$ is a set of non-overlapping, oriented, $k$-dimensional simplices in $K$. For any $\alpha \in[0,1]$, define the $\mathbf{M}_{k}^{\alpha}$ mass of $P$ to be

$$
\mathbf{M}_{k}^{\alpha}(P):=\sum\left|g_{i}\right|^{\alpha} \mathcal{H}^{k}\left(\sigma_{i}\right)
$$

where $\mathcal{H}^{k}$ is the $k$-dimensional Hausdorff measure. We simply write $\mathbf{M}^{\alpha}(P)$ when the dimension is understood from context.

Definition 2.2. Define the flat- $\alpha$ norm of a polyhedral chain $P \in \mathcal{P}_{k}(K)$ by

$$
\mathbf{F}^{\alpha}(P):=\inf \left\{\mathbf{M}_{k}^{\alpha}(P-\partial R)+\mathbf{M}_{k+1}^{\alpha}(R): R \in \mathcal{P}_{k+1}(K)\right\}
$$




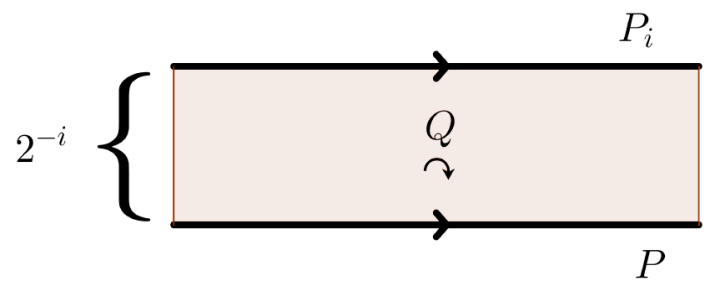

Figure 3. Topology induced by the $\mathbf{M}^{\alpha}$ norm vs. the flat- $\alpha$ norm

or equivalently,

$$
\mathbf{F}^{\alpha}(P):=\inf \left\{\mathbf{M}_{k}^{\alpha}(S)+\mathbf{M}_{k+1}^{\alpha}(R): P=S+\partial R \text { for } S \in \mathcal{P}_{k}(K), R \in \mathcal{P}_{k+1}(K)\right\}
$$

where $\partial$ is the usual boundary operation on polyhedral chains. The flat- $\alpha$ distance between $P_{1}, P_{2} \in \mathcal{P}_{k}(K)$ is then defined to be $\mathbf{F}^{\alpha}\left(P_{1}-P_{2}\right)$.

Most properties are straightforward to prove that the flat- $\alpha$ norm is indeed a norm on $\mathcal{P}_{k}(K)$; the only property not straightforward is that $\mathbf{F}^{\alpha}(P)=0$ implies that $P=0$, which will follow after results in Section 2.6. (See Rem. 2.25.) Also, note that $\mathbf{F}^{\alpha}(\partial P) \leq \mathbf{F}^{\alpha}(P)$ by definition and considering if $P=S+\partial R$, then $\partial P=\partial S$.

At first glance, it may seem more natural to consider the topology induced by the $\mathbf{M}^{\alpha}$ mass instead of creating the flat- $\alpha$ norm. However, the following example elucidates why the flat- $\alpha$ norm is preferable: it more accurately captures the concept of geometric closeness for chains.

Example 2.3. Consider polyhedral chains $\left\{P_{i}\right\}$ and $P$ each consisting of a directed edge of length 1 and weight 1. Suppose that each $P_{i}$ is parallel to $P$ and at (Euclidean) distance $2^{-i}$ from $P$. Since $\mathbf{M}^{\alpha}\left(P_{i}-P\right)=2$ for every $i \geq 0$, the sequence $\left\{P_{i}\right\}$ fails to converge to $P$ in the topology induced by the $\mathbf{M}^{\alpha}$ norm. However, the sequence does converge to $P$ in the flat- $\alpha$ norm since

$$
\mathbf{F}^{\alpha}\left(P_{i}-P\right) \leq \mathbf{M}^{\alpha}\left(P_{i}-P-\partial Q\right)+\mathbf{M}^{\alpha}(Q)=2^{-i+1}+2^{-i}
$$

where $Q$ is the two-dimensional chain with weight 1 as in Figure 3.

An additional benefit of the flat- $\alpha$ norm is the lower semicontinuity of the $\mathbf{M}^{\alpha}$ mass property:

Lemma 2.4. [20, 38] On $\mathcal{P}_{k}(K)$, the $\mathbf{M}^{\alpha}$ mass is lower semicontinuous with respect to the flat- $\alpha$ norm.

Note that Fleming's argument in [20] is extended in White's paper [38] to prove this lemma for polyhedral chains with coefficients in a more generalized normed abelian group $G$; so the above lemma follows from White's results by considering the chain coefficients as elements of the group $\mathbb{R}$ with group norm $|g|^{2}|g|^{\alpha}$ as White notes as an example in [38].

Using the flat- $\alpha$ completion of $\mathcal{P}_{k}(K)$, we may now define general flat chains.

Definition 2.5. Denote by $\mathcal{F}_{k}(K)$ the $\mathbf{F}^{\alpha}$-completion of $\mathcal{P}_{k}(K)$ and call its elements real-valued, $k$-dimensional flat chains. Consider a sequence $\left\{P_{i}\right\} \subset \mathcal{P}_{k}(K)$ converging to $T$ in the flat- $\alpha$ norm and note that

$$
\mathbf{F}^{\alpha}\left(\partial P_{i}-\partial P_{j}\right)=\mathbf{F}^{\alpha}\left(\partial\left(P_{i}-P_{j}\right)\right) \leq \mathbf{F}^{\alpha}\left(P_{i}-P_{j}\right)
$$

Thus, $\left\{\partial P_{i}\right\}$ is a convergent sequence of $(k-1)$-dimensional polyhedral chains and we say that its limit is a $(k-1)$-dimensional flat chain called the boundary of $T$, denoted $\partial T$. 
Remark 2.6. As in [38], one can then define $\mathcal{F}_{k}\left(\mathbb{R}^{m}\right)$ as the union of $\mathcal{F}_{k}(K)$ over all compact sets $K \subset \mathbb{R}^{m}$, resulting in the fact that every real flat chain in $\mathbb{R}^{m}$ is compactly supported. However, we only need to consider chains defined on compact sets for the construction of the flow as well as the application to transport networks.

In order to preserve the lower semicontinuity property of mass, we define the $\mathbf{M}^{\alpha}$ mass of a real-valued flat chain as follows.

Definition 2.7. For any real flat chain $T \in \mathcal{F}_{k}(K)$, define its $\mathbf{M}_{k}^{\alpha}$ mass to be

$$
\mathbf{M}_{k}^{\alpha}(T):=\inf \left\{\liminf _{i \rightarrow \infty} \mathbf{M}_{k}^{\alpha}\left(P_{i}\right):\left\{P_{i}\right\} \stackrel{\mathbf{F}^{\alpha}}{\longrightarrow} T\right\}
$$

We write $\mathbf{M}^{\alpha}(T)$ when the dimension is understood from context.

This definition indeed coincides with the one above when $T$ is polyhedral due to the lower semicontinuity of the $\mathbf{M}^{\alpha}$ mass on $\mathcal{P}_{k}(K)$ with respect to the flat- $\alpha$ norm for polyhedral chains.

Definition 2.8. And in general, we may extend the flat- $\alpha$ norm to real-valued, $k$-dimensional flat chain $T$ by defining

$$
\mathbf{F}^{\alpha}(T):=\inf \left\{\mathbf{M}_{k}^{\alpha}(T-\partial R)+\mathbf{M}_{k+1}^{\alpha}(R): R \in \mathcal{F}_{k+1}(K)\right\}
$$

or equivalently,

$$
\mathbf{F}^{\alpha}(T):=\inf \left\{\mathbf{M}_{k}^{\alpha}(S)+\mathbf{M}_{k+1}^{\alpha}(R): T=S+\partial R \text { for } S \in \mathcal{F}_{k}(K), R \in \mathcal{F}_{k+1}(K)\right\} .
$$

For convenience, we define $\mathbf{N}^{\alpha}(T):=\mathbf{M}_{k}^{\alpha}(T)+\mathbf{M}_{k-1}^{\alpha}(\partial T)$ for $T \in \mathcal{F}_{k}(K)$; and let $\mathcal{N}_{k}^{\alpha}(K):=\left\{T \in \mathcal{F}_{k}(K)\right.$ : $\left.\mathbf{N}^{\alpha}(T)<\infty\right\}$, the set of all real-valued, $k$-dimensional flat chains with both finite $\mathbf{M}^{\alpha}$ mass and finite $\mathbf{M}^{\alpha}$ boundary mass.

Once again, most properties are straightforward to verify to prove that this extended definition of the flat- $\alpha$ norm is indeed a norm on $\mathcal{F}_{k}(K)$ (except for that discussed in Rem. 2.25). We also still have the property that $\mathbf{F}^{\alpha}(\partial T) \leq \mathbf{F}^{\alpha}(T)$.

This next lemma on lower semicontinuity follows from the definition of the $\mathbf{M}^{\alpha}$ mass, considering again the chain coefficients as elements of the group $\mathbb{R}$ with group norm $|g|=|g|^{\alpha}$ and shown in $[20,38]$.

Lemma 2.9. [20, 38] On $\mathcal{F}_{k}(K)$, the $\mathbf{M}^{\alpha}$ mass is lower semicontinuous with respect to the flat- $\alpha$ norm.

Comparing the flat- $\alpha$ norm and the norm induced by the $\mathbf{M}^{\alpha}$ mass on real-valued flat chains, one finds that convergence in the $\mathbf{M}^{\alpha}$ norm is stronger than that in the flat- $\alpha$ norm when $k<m$ since $\mathbf{F}^{\alpha}(T) \leq \mathbf{M}^{\alpha}(T)$. Example 2.3 provides an instance where convergence in the flat- $\alpha$ norm does not imply convergence in the $\mathbf{M}^{\alpha}$ norm. Thus, we will use the flat- $\alpha$ norm to measure closeness of two chains since it takes into consideration the $\mathbf{M}^{\alpha}$ mass of the chains as well as their geometric nearness in $K \subset \mathbb{R}^{m}$.

An additional convenient property of the definitions of flat chains is the following lemma, which says that there exist polyhedral chains $P_{i}$ converging to $T$ in the flat- $\alpha$ norm such that $\mathbf{M}^{\alpha}(T)=\lim _{i \rightarrow \infty} \mathbf{M}^{\alpha}\left(P_{i}\right)$. The proof of this lemma follows from the definition of the $\mathbf{M}^{\alpha}$ mass of a flat chain as well as results from Section 2.6. (See Rem. 2.25.)

Lemma 2.10. $[9,38]$ Let $\alpha \in[0,1]$ and $K \subset \mathbb{R}^{m}$. For $T \in \mathcal{N}_{k}^{\alpha}(K)$, there exist $k$-dimensional polyhedral chains in $K,\left\{P_{i}\right\}$, such that the $P_{i}$ converge to $T$ in the flat- $\alpha$ norm and

$$
\mathbf{M}^{\alpha}\left(P_{i}\right) \rightarrow \mathbf{M}^{\alpha}(T) \quad \text { and } \quad \mathbf{M}^{\alpha}\left(\partial P_{i}\right) \rightarrow \mathbf{M}^{\alpha}(T) .
$$




\subsection{Compactness}

Recall that Fleming defined flat chains and the flat- $\alpha$ norm in order to have the necessary compactness property for employing the direct method of calculus of variations. Using a deformation theorem shown in [37], White proved a more generalized compactness theorem for chains with coefficients in abelian groups with certain properties in the appendix of [40], which we provide for convenience below:

Theorem 2.11. [40] The set of flat chains of finite generalized mass and boundary mass with coefficients in an abelian group $G$ is compact with respect to the generalized flat norm if and only if $G$ has the property that balls are compact in $G$; i.e. $\{g \in G:|g| \leq r\}$ is compact for all $r>0$.

The following corollary immediately follows as it is merely a rephrasing of this theorem to fit our context of real-valued flat chains. That is, we consider $G=\mathbb{R}$ with metric $|g|^{\alpha}$ for $\alpha \in(0,1]$. (Note that balls are indeed compact in $\mathbb{R}$ with this metric.)

Corollary 2.12. [40] Let $\alpha \in(0,1]$. Suppose $\left\{T_{j}\right\} \subset \mathcal{F}_{k}(K)$ with $\sup _{j \geq 1} \mathbf{N}^{\alpha}\left(T_{j}\right)<\infty$. Then there exists a subsequence of $\left\{T_{j}\right\}$ converging with respect to the flat- $\alpha$ norm to a real flat chain $T \in \mathcal{F}_{k}(K)$.

Remark 2.13. Compactness fails in the case $\alpha=0$ since $\left\{g \in \mathbb{R}:|g|^{0} \leq r\right\}=\mathbb{R}$ for all $r \geq 1$. Since we will employ the direct method of calculus of variations at several points in the construction of the flow, we will often refer to Corollary 2.12, and consequently, the $\mathbf{M}^{\alpha}$ reducing flow will be restricted to $\alpha \in(0,1]$.

\subsection{Cartesian products of flat chains}

Note that most of this material on Cartesian products can be found in Fleming's paper developing the theory of flat chains over a finite coefficient group [20], but we include for convenience this development of Cartesian products of real flat chains (with coefficient group with norm $|g|^{\alpha}$ for $\alpha \in[0,1]$ ).

In this paper, we will need the Cartesian product of flat chains to create a sequence of ("birthday cake shaped") higher dimensional chains in $\mathbb{R}^{+} \times \mathbb{R}^{m}$ within the construction of the flow (in Sect. 3.2). While we cannot give a satisfactory definition for the Cartesian product of two arbitrary flat chains, we may provide one for when at least one of the chains is in $\mathcal{N}_{k}^{\alpha}(K)$, which is all we need for the construction of the flow. To do so, we must first define the Cartesian product of two polyhedral chains and then give a bound on the flat- $\alpha$ norm of their Cartesian product.

Definition 2.14. Suppose $P$ is a $k$-dimensional polyhedral chain in $K_{1} \subset \mathbb{R}^{m}$ and $Q$ is an $\ell$-dimensional polyhedral chain in $K_{2} \subset \mathbb{R}^{n}$. Their Cartesian product, denoted $P \times Q$, is a $(k+\ell)$-dimensional polyhedral chain in $K_{1} \times K_{2} \subset \mathbb{R}^{m+n}$.

Note that

$$
\mathbf{M}_{k+\ell}^{\alpha}(P \times Q)=\mathbf{M}_{k}^{\alpha}(P) \mathbf{M}_{\ell}^{\alpha}(Q)
$$

since for $\alpha \in[0,1],\left|g_{i} g_{j}\right|^{\alpha}=\left|g_{i}\right|^{\alpha}\left|g_{j}\right|^{\alpha}$ for $\left\{g_{i}\right\}$ and $\left\{g_{j}\right\}$ the sets of real coefficients for the polyhedral chains $P$ and $Q$, respectively. Moreover,

$$
\partial(P \times Q)=\partial P \times Q+(-1)^{k} P \times \partial Q
$$

To define the Cartesian product in more generality, we will need the following lemma and Lemma 2.10.

Lemma 2.15. [20] Let $\alpha \in[0,1]$. For $P$ and $Q$ as above, $\mathbf{F}^{\alpha}(P \times Q) \leq \mathbf{N}^{\alpha}(P) \mathbf{F}^{\alpha}(Q)$ and likewise, $\mathbf{F}^{\alpha}(P \times Q) \leq$ $\mathbf{F}^{\alpha}(P) \mathbf{N}^{\alpha}(Q)$.

Proof. Here, we will replicate the proof found in Fleming's paper [20] for the reader's convenience. 
Suppose $Q=U+\partial V$ for $U \in \mathcal{P}_{\ell}\left(K_{2}\right)$ and $V \in \mathcal{P}_{\ell+1}\left(K_{2}\right)$. We wish to consider the flat- $\alpha$ norm of $P \times Q=$ $P \times(U+\partial V)$. First, we will show that $\mathbf{F}^{\alpha}(P \times \partial V) \leq \mathbf{N}^{\alpha}(P) \mathbf{M}^{\alpha}(V)$. Note that by equation (2.2),

$$
P \times \partial V= \pm[\partial P \times V-\partial(P \times V)]
$$

Thus, by definition of the flat- $\alpha$ norm, we have

$$
\mathbf{F}^{\alpha}(P \times \partial V) \leq \mathbf{M}^{\alpha}(\partial P \times V)+\mathbf{M}^{\alpha}(P \times V) \leq \mathbf{N}^{\alpha}(P) \mathbf{M}^{\alpha}(V),
$$

using equation (2.1).

So, we have, again using equation (2.1),

$$
\begin{aligned}
\mathbf{F}^{\alpha}(P \times Q) & \leq \mathbf{F}^{\alpha}(P \times U)+\mathbf{F}^{\alpha}(P \times \partial V), \\
& \leq \mathbf{M}^{\alpha}(P \times U)+\mathbf{F}^{\alpha}(P \times \partial V), \\
& \leq \mathbf{M}^{\alpha}(P) \mathbf{M}^{\alpha}(U)+\mathbf{N}^{\alpha}(P) \mathbf{M}^{\alpha}(V), \\
& \leq \mathbf{N}^{\alpha}(P)\left(\mathbf{M}^{\alpha}(U)+\mathbf{M}^{\alpha}(V)\right),
\end{aligned}
$$

where the last inequality follows from the fact that $\mathbf{M}^{\alpha}(P) \leq \mathbf{M}^{\alpha}(P)+\mathbf{M}^{\alpha}(\partial P)=\mathbf{N}^{\alpha}(P)$.

Since we showed the above for all $U \in \mathcal{P}_{\ell}\left(K_{2}\right)$ and $V \in \mathcal{P}_{\ell+1}\left(K_{2}\right)$ such that $Q=U+\partial V$, we must have $\mathbf{F}^{\alpha}(P \times Q) \leq \mathbf{N}^{\alpha}(P) \mathbf{F}^{\alpha}(Q)$, as desired.

Definition 2.16. Now we may define the Cartesian product of $T \in \mathcal{N}_{k}^{\alpha}\left(K_{1}\right)$ for compact set $K_{1} \subset \mathbb{R}^{m}$ and $S \in \mathcal{F}_{\ell}\left(K_{2}\right)$ for compact set $K_{2} \subset \mathbb{R}^{n}$. We first define $T \times Q$ for any $Q \in \mathcal{P}_{\ell}\left(K_{2}\right)$ as follows. Let $\left\{P_{j}\right\} \subset \mathcal{P}_{k}\left(K_{1}\right)$ converge to $T$ in the flat- $\alpha$ norm on $\mathcal{F}_{k}\left(K_{1}\right)$ so that $\mathbf{N}^{\alpha}\left(P_{j}\right) \rightarrow \mathbf{N}^{\alpha}(T)$. (Lem. 2.10 shows that there exists such a sequence.) Then by Lemma 2.15 , we have for any $Q \in \mathcal{P}_{\ell}\left(K_{2}\right)$

$$
\mathbf{F}^{\alpha}\left[\left(P_{i} \times Q\right)-\left(P_{j} \times Q\right)\right]=\mathbf{F}^{\alpha}\left[\left(P_{i}-P_{j}\right) \times Q\right] \leq \mathbf{F}^{\alpha}\left(P_{i}-P_{j}\right) \mathbf{N}^{\alpha}(Q) .
$$

So, $\left\{P_{j} \times Q\right\} \subset \mathcal{P}_{k+\ell}\left(K_{1} \times K_{2}\right)$ converges in the flat- $\alpha$ norm on $\mathcal{F}_{k+\ell}\left(K_{1} \times K_{2}\right)$ to the real-valued, $k+\ell$ dimensional flat chain $T \times Q$. For our purposes, this definition of a Cartesian product of a flat chain and a polygonal chain is sufficient; however, we include the following discussion to define the Cartesian product in more generality simply for completeness of exposition.

Now take $\left\{Q_{i}\right\} \subset \mathcal{P}_{\ell}\left(K_{2}\right)$ converging to $S \in \mathcal{F}_{\ell}\left(K_{2}\right)$ in the flat- $\alpha$ norm on $\mathcal{F}_{\ell}\left(K_{2}\right)$. Since we have $\mathbf{N}^{\alpha}\left(P_{j}\right) \rightarrow$ $\mathbf{N}^{\alpha}(T)$, we can extend the result of Lemma 2.15 to real-valued, $k$-dimensional flat chain $T$ in place of the polyhedral chain $P$. Note that then

$$
\mathbf{F}^{\alpha}\left[T \times\left(Q_{i}-Q_{j}\right)\right] \leq \mathbf{N}^{\alpha}(T) \mathbf{F}^{\alpha}\left(Q_{i}-Q_{j}\right) \rightarrow 0
$$

because $\mathbf{N}^{\alpha}(T)<\infty$ by assumption and because $\left\{Q_{i}\right\} \subset \mathcal{P}_{\ell}\left(K_{2}\right)$ converges to $S \in \mathcal{F}_{\ell}\left(K_{2}\right)$ in the flat- $\alpha$ norm on $\mathcal{F}_{\ell}\left(K_{2}\right)$. That is, we have a convergent sequence of real-valued, $k+\ell$ dimensional flat chains $\left\{T \times Q_{i}\right\}$ converging in the flat- $\alpha$ norm on $\mathcal{F}_{k+\ell}\left(K_{1} \times K_{2}\right)$ to $T \times S \in \mathcal{F}_{k+\ell}\left(K_{1} \times K_{2}\right)$. Note that, for this sequence to converge, we indeed needed $\mathbf{N}^{\alpha}(T)<\infty$.

We have similar properties for this more generalized definition of Cartesian product of flat chains as we had for polygonal ones. Let $T \in \mathcal{N}_{k}^{\alpha}\left(K_{1}\right)$ for compact set $K_{1} \subset \mathbb{R}^{m}$ and $S \in \mathcal{F}_{\ell}\left(K_{2}\right)$ for compact set $K_{2} \subset \mathbb{R}^{n}$. We have the operation $\times$ is bilinear and

$$
\partial(T \times S)=\partial T \times S+(-1)^{k} T \times \partial S,
$$




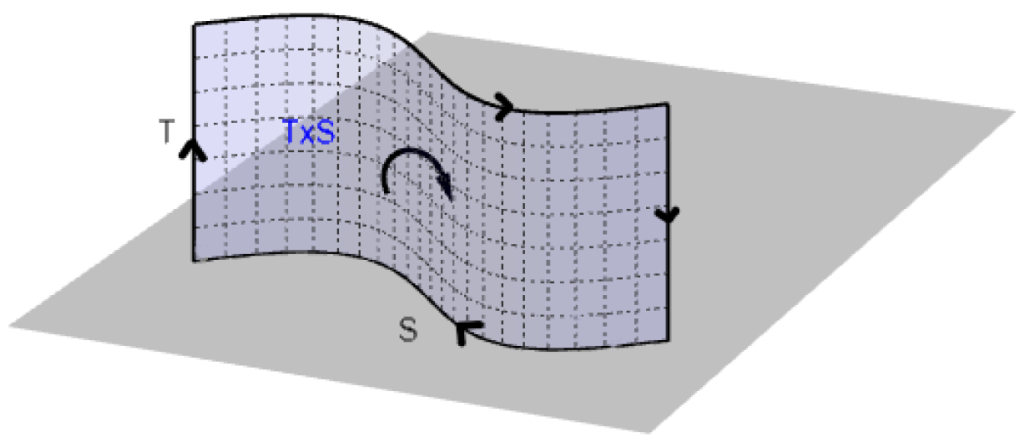

Figure 4. Example of the Cartesian product of $T \times S$ where $T=[|r, t|]$ for $r<t$ and $S$ is a 1-dimensional chain in $\mathbb{R}^{2}$

which can be seen in the example of Figure 4. Also, we have preserved $\mathbf{M}_{k+\ell}^{\alpha}(T \times S) \leq \mathbf{M}_{k}^{\alpha}(T) \mathbf{M}_{\ell}^{\alpha}(S)$ and $\mathbf{F}^{\alpha}(T \times S) \leq \mathbf{N}^{\alpha}(T) \mathbf{F}^{\alpha}(S)$.

Recall that within the construction of the flow (in Sect. 3.2), we will need the Cartesian product to create a sequence of ("birthday cake shaped") higher dimensional chains in $\mathbb{R}^{+} \times \mathbb{R}^{m}$. (See Fig. 5.) So, we are particularly interested in the case with $S \in \mathcal{F}_{\ell}\left(K_{2}\right)$ for compact set $K_{2} \subset \mathbb{R}^{n}$ as before and for $r<t, T=[|r, t|] \in \mathcal{N}_{1}^{\alpha}\left(K_{1}\right)$ for $K_{1} \subset \mathbb{R}$ compact, where [| $\left.\cdot \mid\right]$ denotes a positively oriented, polygonal chain of appropriate dimension with weight 1 . Thus, $T=[|r, t|]$ is the interval $(r, t)$ considered as a positively oriented, polygonal chain of weight 1 . Because the flow construction relies on this special case, we will explicitly explore the above discussion in this context. Here, $[|r, t|] \times S \in \mathcal{F}_{1+\ell}\left(K_{1} \times K_{2}\right)$ and its boundary is

$$
\partial([|r, t|] \times S)=[|t|] \times S-[|r|] \times S-[|r, t|] \times \partial S \subset \mathcal{F}_{\ell}\left(K_{1} \times K_{2}\right),
$$

by the bilinearity of $\times$, equation (2.3), $\partial[|r, t|]=[|t|]-[|s|]$, and the dimension of $T$ being 1. (Above, $[|t|],[|r|]$ are points considered as positively oriented, zero dimensional chains of weight 1.) Additionally,

$$
\mathbf{M}_{1+\ell}^{\alpha}([|r, t|] \times S)=(t-r) \mathbf{M}_{\ell}^{\alpha}(S)
$$

which also leads to

$$
\begin{aligned}
\mathbf{M}_{\ell}^{\alpha}(\partial([|r, t|] \times S)) & =\mathbf{M}_{\ell}^{\alpha}([|t|] \times S-[|r|] \times S-[|r, t|] \times \partial S), \\
& =2 \mathbf{M}_{\ell}^{\alpha}(S)+(t-r) \mathbf{M}_{\ell-1}^{\alpha}(\partial S)
\end{aligned}
$$

Example 2.17. The above equations can be further exemplified using Figure 4 depicting $[|r, t|] \times S$ for $S \in$ $\mathcal{F}_{1}\left(K_{2}\right)$ for compact set $K_{2} \subset \mathbb{R}^{2}$, say with weight 2 . In this example, $[|t|] \times S$ is the 1-dimensional chain parallel to $S$ on the top of the diagram with orientation indicated by the arrow, $-[|r|] \times S$ is the 1-dimensional chain on the bottom of the diagram with indicated orientation, and $-[|r, t|] \times \partial S$ are the 1-dimensional chains parallel to $T$ on the left and right of the diagram all with weight 2 . Then, $\mathbf{M}^{\alpha}([|r, t|] \times S)$ measures the weighted area shaded in blue and $\mathbf{M}^{\alpha}(\partial([|r, t|] \times S))$ measures the weighted length of the boundary.

\subsection{Push forward of real flat chains by Lipschitz maps}

Since the construction of the flow will require a "birthday cake" decomposition, we will need finite $\mathbf{M}^{\alpha}$ mass cycles to bound higher dimensional chains achieving the considered norm. For integral chains (i.e., chains with integer coefficients), this fact would hold for the flat- $\alpha$ norm because of the isoperimetric inequality [8]. However, real-valued chains do not possess an isoperimetric property. So, we will define a new norm, called the fill- $\alpha$ norm, using cones over chains. Then, we will show that finite cycles bound higher dimensional chains 
achieving this new norm. In order to define cones over chains, we will need to first define push forwards of real-valued chains and give the homotopy formula, which we do now. Moreover, we will need push forwards of chains under projection maps, so we will be particularly interested in Lipschitz maps in the following sections.

If $g: \mathbb{R}^{m} \rightarrow \mathbb{R}^{n}$ is a piecewise linear map, then it induces a chain homomorphism $g_{\sharp}: \mathcal{P}_{*}\left(K_{1}\right) \rightarrow \mathcal{P}_{*}\left(K_{2}\right)$ for compact sets $K_{1} \subset \mathbb{R}^{m}$ and $K_{2} \subset \mathbb{R}^{n}$, and where $*$ represents any chain dimension as in [38]. This map is defined by

$$
g_{\sharp}: \sum a_{i} \sigma_{i} \mapsto \sum a_{i} g\left(\sigma_{i}\right),
$$

so that $g_{\sharp}$ respects the chain grading, $g_{\sharp}(S+T)=g_{\sharp} S+g_{\sharp} T$ for $S, T \in \mathcal{P}_{*}\left(K_{1}\right)$, and $g$ commutes with the boundary map $\partial$. For simplified notation, we write $g_{\sharp} T$ to represent the image of $T$ under $g_{\sharp}$. This map on polyhedral chains may be extended uniquely to real flat chains if $g_{\sharp}$ is Lipschitz with respect to the $\mathbf{M}^{\alpha}$ norm:

Lemma 2.18. [9] Suppose $g_{\sharp}: \mathcal{P}_{*}\left(K_{1}\right) \rightarrow \mathcal{P}_{*}\left(K_{2}\right)$ for compact sets $K_{1} \subset \mathbb{R}^{m}$ and $K_{2} \subset \mathbb{R}^{n}$ is a chain homomorphism where * represents any chain dimension. Also, suppose $g_{\sharp}$ is Lipschitz with respect to the $\mathbf{M}^{\alpha}$ norm; i.e., $\mathbf{M}^{\alpha}\left(g_{\sharp} T\right) \leq \mathbf{L i p}\left(g_{\sharp}\right)^{k} \mathbf{M}^{\alpha}(T)$ for $T \in \mathcal{P}_{k}\left(K_{1}\right)$. Then, $g_{\sharp}$ is also Lipschitz with respect to the flat- $\alpha$ norm consequently inducing a unique extension to $\mathcal{F}_{*}\left(K_{1}\right) \rightarrow \mathcal{F}_{*}\left(K_{2}\right)$.

Proof. Let $T \in \mathcal{F}_{k}\left(K_{1}\right)$. Then since $g_{\sharp}$ is Lipschitz with respect the the $\mathbf{M}^{\alpha}$ norm, we have for $R \in \mathcal{F}_{k+1}\left(K_{1}\right)$,

$$
\mathbf{M}^{\alpha}\left(g_{\sharp}(T-\partial R)\right)+\mathbf{M}^{\alpha}\left(g_{\sharp} R\right) \leq \max \left\{\mathbf{L i p}\left(g_{\sharp}\right)^{k}, \mathbf{L i p}\left(g_{\sharp}\right)^{k+1}\right\}\left(\mathbf{M}^{\alpha}(T-\partial R)+\mathbf{M}^{\alpha}(R)\right) \text {. }
$$

And so, the desired inequality, $\mathbf{F}_{K_{2}}^{\alpha}\left(g_{\sharp}(T)\right) \leq \mathbf{L i p}\left(g_{\sharp}\right) \mathbf{F}_{K_{1}}^{\alpha}(T)$, follows from taking an infimum over all $R \in$ $\mathcal{F}_{k+1}\left(K_{1}\right)$.

Similarly, we may extend a Lipschitz map $f: K_{1} \subset \mathbb{R}^{m} \rightarrow K_{2} \subset \mathbb{R}^{n}$ for compact sets $K_{1}, K_{2}$ to a chain homomorphism $f_{\sharp}: \mathcal{F}_{k}\left(K_{1}\right) \rightarrow \mathcal{F}_{k}\left(K_{2}\right)$ by approximating $f$ by piecewise linear maps, resulting in a similar lemma. The proof of this lemma follows a similar argument as that found in [20].

Lemma 2.19. Let $\alpha \in[0,1]$. If $f: \mathbb{R}^{m} \rightarrow \mathbb{R}^{n}$ is a Lipschitz map, then

$$
\mathbf{M}^{\alpha}\left(f_{\sharp} T\right) \leq(\operatorname{Lip} f)^{k} \mathbf{M}^{\alpha}(T),
$$

and

$$
\mathbf{F}^{\alpha}\left(f_{\sharp} T\right) \leq \max \left\{(\operatorname{Lip} f)^{k},(\operatorname{Lip} f)^{k+1}\right\} \mathbf{F}^{\alpha}(T),
$$

for all $T \in \mathcal{F}_{k}\left(K_{1}\right)$ where $K_{1} \subset \mathbb{R}^{m}$ is compact.

Let $K \subset \mathbb{R}^{m}$ be compact and let $f, g: K \subset \mathbb{R}^{m} \rightarrow \mathbb{R}^{m}$ be smooth mappings. The affine homotopy of $f$ to $g$ is the map $h:[0,1] \times K \rightarrow \mathbb{R}^{m}$, where $h(0, x)=f(x)$ and $h(1, x)=g(x)$ for all $x \in K$. If $T \in \mathcal{F}_{k}(K)$ has compact support and if the restriction of $h$ to $[0,1] \times \operatorname{spt} T$ is proper, then $h_{\sharp}([|(0,1)|] \times T)$ is well-defined and by equation (2.4),

$$
\partial h_{\sharp}([|(0,1)|] \times T)=g_{\sharp} T-f_{\sharp} T-h_{\sharp}([|(0,1)|] \times \partial T) .
$$

Rearranging the above equation gives the homotopy formula:

$$
g_{\sharp} T-f_{\sharp} T=\partial h_{\sharp}([|(0,1)|] \times T)+h_{\sharp}([|(0,1)|] \times \partial T) .
$$




\subsection{Cones over flat chains}

Now that we have defined push forwards of real-valued chains and given the homotopy formula, we can define cones over chains. Essentially, the construction of the flow requires finite $\mathbf{M}^{\alpha}$ mass cycles to bound higher dimensional chains achieving the considered norm, and for integral chains this is shown by an isoperimetric inequality, which is not true for real-valued chains. So, we are working towards Corollary 2.22 in order to define the new needed norm defined in the next section.

Fix $x_{0} \in K$ for $K \subset \mathbb{R}^{m}$ convex, and thus, star-shaped with respect to the point $x_{0}$ (i.e., $t x+(1-t) x_{0} \in K$ for each $x \in K$ and each $0 \leq t \leq 1$.)

Definition 2.20. Let $T \in \mathcal{F}_{k}(K)$ with compact support. Define $h: \mathbb{R} \times K \rightarrow \mathbb{R}^{m}$ by $h(t, x)=t x+(1-t) x_{0}$. Then, the cone over $T$, denoted cone $(T)$, is given by $h_{\sharp}([|(0,1)|] \times T)$.

It directly follows that cone $(T) \in \mathcal{F}_{k+1}(K)$, and by the homotopy formula,

$$
\partial(\operatorname{cone}(T))=T-\operatorname{cone}(\partial T) .
$$

Because of this, every cycle bounds its cone, and the following lemma implies that this chain will also have finite $\mathbf{M}^{\alpha}$ mass if the cycle does.

Lemma 2.21. [9] Let $\alpha \in[0,1]$. If $T \in \mathcal{F}_{k}(K)$ has compact support, then

$$
\mathbf{M}^{\alpha}(\operatorname{cone}(T)) \leq \operatorname{diam}(K) \mathbf{M}^{\alpha}(T) .
$$

Proof. This follows from

$$
\mathbf{M}^{\alpha}(\operatorname{cone}(T)) \leq \mathbf{M}^{\alpha}([0, \operatorname{diam}(K)] \times T)=\operatorname{diam}(K) \mathbf{M}^{\alpha}(T),
$$

by equation (2.5).

Corollary 2.22. Every cycle of finite $\mathbf{M}^{\alpha}$ mass bounds a chain of finite $\mathbf{M}^{\alpha}$ mass. More precisely, if $T \in \mathcal{N}_{k}^{\alpha}(K)$ and $\partial T=0$, then there exists an $S \in \mathcal{F}_{k+1}(K)$ with $\partial S=T$ and $\mathbf{M}^{\alpha}(S)<\infty$; namely, $S=$ cone $(T)$.

In [37], White proves a similar result as the above Corollary to similarly extend Almgren's isoperimetric inequality [1] and Fleming's isoperimetric inequality [20].

\subsection{Defining a filling norm for cycles}

Recall that we need finite $\mathbf{M}^{\alpha}$ mass cycles to bound higher dimensional chains achieving some norm since the construction of the flow requires a "birthday cake" decomposition. Using the above results, we can define this new norm, which we will call the fill- $\alpha$ norm, and show that finite cycles indeed bound higher dimensional chains achieving this norm. While this is a new norm in a sense, it is merely a simplified flat norm for cycles; in fact, the fill- $\alpha$ norm and flat- $\alpha$ norm are equivalent for cycles, and thus, either may be used to capture the idea of "closeness" of two chains with the same boundary. The only instance of such a norm (defined for polyhedral chains) that the author is aware of is in the lecture notes by Chodosh on Brian White's Topics in Geometric Measure Theory course from Spring 2012 [9]. However, this section's results, while necessary for the construction of the flow, do not provide novel insight into real flat chains since the new norm is equivalent to the flat norm on chains.

As before, assume $K \subset \mathbb{R}^{m}$ is convex, and for convenience, denote the cycles in $\mathcal{F}_{k}(K)$ by $\mathcal{Z}_{k}(K)$; i.e.,

$$
\mathcal{Z}_{k}(K):=\left\{T \in \mathcal{F}_{k}(K): \partial T=0\right\}
$$


By the above definition of cone, we know the following norm is well-defined.

Definition 2.23. For $T \in \mathcal{Z}_{k}(K)$ with compact support, define a filling- $\alpha$ mass for $T$ as

$$
\mathbf{F i l l}^{\alpha}(T):=\inf \left\{\mathbf{M}^{\alpha}(Q): \partial Q=T \text { with } \operatorname{spt} Q \subset K\right\} .
$$

The following lemma shows that the fill- $\alpha$ norm and the flat- $\alpha$ norm are equivalent for cycles. The proof follows the same argument found in [9].

Lemma 2.24. For $T \in \mathcal{Z}_{k}(K)$ with finite $\mathbf{M}^{\alpha}$ mass, then

$$
\mathbf{F}^{\alpha}(T) \leq \mathbf{F i l l}^{\alpha}(T) \leq c \mathbf{F}^{\alpha}(T)
$$

where $c$ is a constant depending only on $K \subset \mathbb{R}^{m}$.

Proof. The first inequality follows from the fact that the infimum on the right-hand side is taken over a subset of $(k+1)$-dimensional flat chains while the infimum on the left-hand side is taken over all $(k+1)$-dimensional flat chains. For the second inequality, write $T=Q+\partial R$. Note that $\partial T=\partial Q=0$, and so, $Q=\partial(\operatorname{cone}(Q))$ by equation (2.8). Thus, $T=\partial(\operatorname{cone}(Q)+R)$. By the triangle inequality and Lemma 2.21,

$$
\mathbf{F i l l}^{\alpha}(T) \leq \mathbf{M}^{\alpha}(\operatorname{cone}(Q))+\mathbf{M}^{\alpha}(R) \leq(\operatorname{diam}(K)) \mathbf{M}^{\alpha}(Q)+\mathbf{M}^{\alpha}(R) .
$$

Taking the infimum over all such $Q, R$ proves the second inequality for constant $c:=\max \{\operatorname{diam}(K), 1\}$.

Remark 2.25. This lemma may be used to complete the argument that the flat- $\alpha$ norm is indeed a norm as it may be used to show that $\mathbf{F}^{\alpha}(T)=0$ implies $T=0$. Additionally, it is needed for the proof of Lemma 2.10.

Another consequence of this lemma is that the fill- $\alpha$ norm is lower semicontinuous with respect to the flat- $\alpha$ norm:

Corollary 2.26. On $\mathcal{Z}_{k}(K)$, the Fill $^{\alpha}$ mass is lower semicontinuous with respect to the flat- $\alpha$ norm.

We will need in the flow construction to apply a projection, so we will need a bound on the fill- $\alpha$ norm under a Lipschitz map in addition to Lemma 2.19. That is, we need the following lemma on how a Lipschitz map $f: \mathbb{R}^{m} \rightarrow \mathbb{R}^{n}$ extends to a map on flat cycles that is Lipschitz with respect to the fill- $\alpha$ norm. This lemma follows from the fact that cycles map to cycles $\left(\partial\right.$ and $f_{\sharp}$ commute [19]) and Lemma 2.19.

Lemma 2.27. Let $f: K \subset \mathbb{R}^{m} \rightarrow \mathbb{R}^{n}$ be a Lipschitz map, then

$$
\mathbf{F i l l}^{\alpha}\left(f_{\sharp} T\right) \leq \max \left\{(\operatorname{Lip} f)^{k},(\operatorname{Lip} f)^{k+1}\right\} \mathbf{F i l l}^{\alpha}(T)
$$

for all $T \in \mathcal{Z}_{k}(K)$.

Note that there is a slight complication above in ensuring the fill- $\alpha$ norm on $\mathcal{Z}_{k}(f(K))$ is well-defined on the left hand side of the inequality since $f(K)$ may not be convex; but, you may consider the convex hull without any loss of generality or consider $\mathcal{Z}_{k}\left(\mathbb{R}^{n}\right)$ using Remark 2.6.

Keep in mind that when defining the discrete sequences in the flow, we will use the fill- $\alpha$ norm to capture the idea of "closeness" of two chains with the same boundary (in functional $G$ that will be defined in equation (3.1)). Moreover, for the "birthday cake" decomposition, we will need the existence of a higher dimensional chain achieving the fill- $\alpha$ norm between the two sequential chains forming its boundary, and thus, need the following theorem. Notice that this is restricted to $\alpha \in(0,1]$ because its proof relies on the Compactness Theorem (Thm. 2.12). First, we state a necessary lemma for the proof.

Theorem 2.28. Let $\alpha \in(0,1]$. For every $T \in \mathcal{Z}_{k}(K)$ with finite $\mathbf{M}^{\alpha}$ mass, there exists an $S \in \mathcal{F}_{k+1}(K)$ with $\partial S=T$ and $\mathbf{M}^{\alpha}(S)=$ Fill $^{\alpha}(T)$. 
Proof. From Corollary 2.22, we know $\mathbf{F i l l}^{\alpha}(T)=\inf \left\{\mathbf{M}^{\alpha}(Q): \partial Q=T\right.$ with $\left.\operatorname{spt} Q \in K\right\}<\infty$, and so, we may take a minimizing sequence $\left\{S_{i}\right\} \subset \mathcal{F}_{k+1}(K)$ with $\lim _{i \rightarrow \infty} \mathbf{M}^{\alpha}\left(S_{i}\right)=$ Fill $^{\alpha}(T)$ and $\partial S_{i}=T$ for each $i$. Since $\lim _{i \rightarrow \infty} \mathbf{M}^{\alpha}\left(S_{i}\right)=$ Fill $^{\alpha}(T)<\infty$, the supremum of $\left\{\mathbf{M}^{\alpha}\left(S_{i}\right)\right\}$ must be finite. Thus,

$$
\sup _{i}\left\{\mathbf{N}^{\alpha}\left(S_{i}\right)\right\}=\sup _{i}\left\{\mathbf{M}^{\alpha}\left(S_{i}\right)+\mathbf{M}^{\alpha}(T)\right\}<\infty .
$$

By the Compactness Corollary 2.12, there exists a subsequence of $\left\{S_{i}\right\}$ converging in the flat- $\alpha$ norm to some $S \in \mathcal{F}_{k+1}(K)$ with $\partial S=\lim _{i^{\prime} \rightarrow \infty} \partial S_{i^{\prime}}=T$. Additionally,

$$
\text { Fill }^{\alpha}(T) \leq \mathbf{M}^{\alpha}(S) \leq \lim _{i^{\prime} \rightarrow \infty} \mathbf{M}^{\alpha}\left(S_{i^{\prime}}\right)=\text { Fill }^{\alpha}(T),
$$

by the lower semicontinuity of $\mathbf{M}^{\alpha}$ mass with respect to the flat- $\alpha$ norm (Lem. 2.9).

\subsection{Slicing of real flat chains}

The $\mathbf{M}^{\alpha}$ mass reducing flow will be defined as a real flat chain of finite mass whose slices dictate the flow. Hence, we must define and give some properties for slicing real-valued flat chains. Of particular importance to the $\mathbf{M}^{\alpha}$ mass reducing flow will be Lemma 2.31. For further background in slicing, see De Pauw and Hardt's paper on flat chains in metric spaces [12] and White's paper [38].

Definition 2.29. Let $T \in \mathcal{N}_{k}^{\alpha}(K), t \in \mathbb{R}$, and $f: K \subset \mathbb{R}^{m} \rightarrow \mathbb{R}$ be Lipschitz. Then we may define the slice of $T$ as

$$
\left\langle T, f, t_{+}\right\rangle:=-\partial\left(T\llcorner\{x: f(x)>t\})+(\partial T)\left\llcorner\{x: f(x)>t\} \in \mathcal{F}_{k-1}(K),\right.\right.
$$

where $L$ represents restriction.

Lemma 2.30. [38] Suppose that $\alpha \in(0,1], T \in \mathcal{N}_{k}^{\alpha}(K)$ and $p: \mathbb{R}^{m} \rightarrow \mathbb{R}$ is an orthogonal projection. Then, for $\alpha \in(0,1]$,

$$
\int_{\mathbb{R}} \mathbf{M}^{\alpha}\left(\left\langle T, p, t_{+}\right\rangle\right) \mathrm{d} t \leq \mathbf{M}^{\alpha}(T)
$$

and

$$
\int_{\mathbb{R}} \mathbf{F}^{\alpha}\left(\left\langle T, p, t_{+}\right\rangle\right) \mathrm{d} t \leq \mathbf{F}^{\alpha}(T)
$$

Lemma 2.31. [12] Let $T_{j}, T \in \mathcal{F}_{k}(K)$ with $\sup _{j}\left\{\mathbf{N}^{\alpha}\left(T_{j}\right)\right\}<\infty$ and suppose that the sequence $\left\{T_{j}\right\}$ converges to $T$ in the flat- $\alpha$ norm. Let $p: \mathbb{R}^{m} \rightarrow \mathbb{R}$ be an orthogonal projection. Then, for almost every $t \in \mathbb{R}$, there is a subsequence $j^{\prime}$ such that

$$
\sum_{j^{\prime}=1}^{\infty} \mathbf{F}^{\alpha}\left(\left\langle T_{j^{\prime}}-T, p, t_{+}\right\rangle\right)<\infty
$$

Hence,

$$
\lim _{j^{\prime} \rightarrow \infty} \mathbf{F}^{\alpha}\left(\left\langle T_{j^{\prime}}, p, t_{+}\right\rangle-\left\langle T, p, t_{+}\right\rangle\right)=0
$$


i.e., the slices of the $T_{j^{\prime}}$ converge in the flat- $\alpha$ norm to the slice of $T$.

Proof. The main pieces of the argument presented in [12] include first passing to a subsequence to ensure that $\sum_{j^{\prime}=1}^{\infty} \mathbf{F}^{\alpha}\left(T_{j^{\prime}}-T\right)<\infty$, and then, the result follows from the fact that under these conditions

$$
\int_{\mathbb{R}} \sum_{j^{\prime}=1}^{\infty} \mathbf{F}^{\alpha}\left(\left\langle T_{j^{\prime}}-T, p, t_{+}\right\rangle\right) \mathrm{d} t=\sum_{j^{\prime}=1}^{\infty} \int_{\mathbb{R}} \mathbf{F}^{\alpha}\left(\left\langle T_{j^{\prime}}-T, p, t_{+}\right\rangle\right) \mathrm{d} t
$$

and Lemma 2.30.

Finally, the following corollary results from the above lemma and lower semicontinuity of the $\mathbf{M}^{\alpha}$ mass (Lem. 2.9).

Corollary 2.32. [12] Under the same assumptions in Lemma 2.31, we have

$$
\mathbf{M}^{\alpha}\left(\left\langle T, p, t_{+}\right\rangle\right) \leq \liminf _{j^{\prime} \rightarrow \infty} \mathbf{M}^{\alpha}\left(\left\langle T_{j^{\prime}}, p, t_{+}\right\rangle\right)
$$

for almost every $t \in \mathbb{R}$.

\section{THE FLOW}

In this section, we construct an $\mathbf{M}^{\alpha}$ mass reducing flow for real-valued flat chains. That is, the entirety of Section 3 constitutes the proof of the main theorem of this paper, Theorem 3.1, by giving the construction of this flow. For the rest of the paper, we will always assume that $\alpha \in(0,1]$ and that $K \subset \mathbb{R}^{m}$ is compact and convex.

Theorem 3.1. For any fixed $C \in \mathbb{R}^{+}$and any $T_{0} \in \mathcal{N}_{k}^{\alpha}(K)$, a real-valued flat chain with compact support contained in some compact, convex set $K \subset \mathbb{R}^{m}$, there exists a flow giving real-valued, $k$-dimensional flat chains $T_{t}$ for almost every $t \in[0, C]$ so that $\partial T_{t}=\partial T_{0}$, and

$$
\mathbf{M}^{\alpha}\left(T_{t}\right) \leq \mathbf{M}^{\alpha}\left(T_{0}\right) .
$$

Additionally,

$$
\mathbf{F}^{\alpha}\left(T_{r}-T_{t}\right) \leq(r-t) \mathbf{M}^{\alpha}\left(T_{0}\right)
$$

for almost every $0 \leq t<r \leq C$.

The above $T_{t}$ will be slices of a higher dimensional chain found by taking a limit of "birthday cake" decompositions created out of discrete sequences of real-valued flat chains. In each sequence, each chain minimizes a functional that tends to reduce $\mathbf{M}^{\alpha}$ mass while ensuring that each chain stays geometrically close to the previous one in the sequence. Note that this flow is interesting for chains $T_{0}$ not a local minimum of this functional, as we'll see in examples.

While the proof of this theorem is this entire section of the paper, we briefly reiterate the outline of the construction here in more detail for convenience of the reader. First, we will find step minimizing sequences of chains in Section 3.1, and out of these sequences, we will create "birthday cake" decompositions, $S_{h}$ 's, at the beginning of Section 3.2. Then, we will take a limit of these higher dimensional chains to find real flat chain $S$ in Theorem 3.6. The "time slices" $T_{t}$ of $S$ are the chains mentioned above in Theorem 3.1. Theorems 3.7 and 3.8 will show that the flow dictated by these $T_{t}$ indeed possess both the mass decreasing property and the Lipschitz continuous with respect to the flat- $\alpha$ norm property stated in Theorem 3.1.

A helpful analogy for intuition behind this construction is children's flip books and animations. Essentially, the discrete minimizing sequence of chains can be considered as pages of a flip book, while the higher dimensional 
chains $S_{h}$ can be conceptualized as sewing these pages together into a flip book. When flipping through this flip book, one would see a sequence of chains depicted moving from an original chain to a locally optimal one in such a way that preserves boundary. The smaller the $h$, the "smoother" this movement would appear when flipping through the book because the drawing on the subsequent page will only be a slight variation of the previous. Then, $S$ would represent the limit of these flip books (taking $h \rightarrow 0$ ) resulting in a "smooth" looking animation having time stamp depicting $T_{t}$ at time $t$. The result is a "nice" animation flowing from the original chain to an $\mathbf{M}^{\alpha}$ locally optimal one in such a way that preserves the boundary of the original.

\subsection{Step minimizing sequence}

We begin by creating the discrete minimizing sequence of chains for each fixed step size. Recall that we will always assume that $\alpha \in(0,1]$ and that $K \subset \mathbb{R}^{m}$ is compact and convex. We will begin with a fixed step size $h>0$ and let $T_{0} \in \mathcal{N}_{k}^{\alpha}(K)$ have compact support. We will then inductively define a sequence of real-valued flat chains $\left\{T_{j}^{h}\right\}$ so that $T_{0}^{h}=T_{0}$ and $T_{j}^{h}$ (step with respect to $h$ ) minimizes a functional that reduces the $\mathbf{M}^{\alpha}$ mass of a chain; however, we will require that this sequence keeps the boundary fixed as well as ensuring that the next chain is geometrically close to the previous, by including a term involving the fill- $\alpha$ mass in the functional. To do this, we will need to define the following functional which has two terms: one involving the $\mathbf{M}^{\alpha}$ mass and the other involving the fill- $\alpha$ mass. The following theorem states that there exists a minimizer to this functional, which we will then apply to each $H=T_{j}^{h}$ to construct our discrete minimizing sequence of step size $h$.

Given $H \in \mathcal{N}_{k}^{\alpha}(K)$ with compact support in $K$, define

$$
G_{H}(T):=\left[\mathbf{M}^{\alpha}(T)\right]^{2}+\frac{1}{h}\left[\mathbf{F i l l}{ }^{\alpha}(H-T)\right]^{2} .
$$

Theorem 3.2. For each $H \in \mathcal{N}_{k}^{\alpha}(K)$ with compact support in $K$, there exists some chain achieving $\min G_{H}(T)$ among all $T \in \mathcal{N}_{k}^{\alpha}(K)$ with compact support in $K$ and $\partial T=\partial H$.

Proof. Let $H \in \mathcal{N}_{k}^{\alpha}(K)$. Consider the collection of real flat chains

$$
\mathcal{C}=\left\{T: T \in \mathcal{N}_{k}^{\alpha}(K) \text { with compact support in } K \text { and } \partial T=\partial H\right\}
$$

which is non-empty since $H \in \mathcal{C}$. Set

$$
L:=\inf \left\{G_{H}(T): T \in \mathcal{C}\right\}
$$

which is finite because $H \in \mathcal{C}$. Also let $\left\{R_{i}\right\} \subset \mathcal{C}$ be a minimizing sequence such that

$$
\lim _{i \rightarrow \infty} G_{H}\left(R_{i}\right)=L
$$

then, $\sup _{i} G_{H}\left(R_{i}\right)<\infty$.

Because of this and since $\left\{R_{i}\right\} \subset \mathcal{C}$, we have

$$
\begin{aligned}
\sup _{i}\left\{\mathbf{N}^{\alpha}\left(R_{i}\right)\right\} & =\sup _{i}\left\{\mathbf{M}^{\alpha}\left(R_{i}\right)+\mathbf{M}^{\alpha}\left(\partial R_{i}\right)\right\}, \\
& \leq \sup _{i}\left\{\sqrt{G_{H}\left(R_{i}\right)}+\mathbf{M}^{\alpha}(\partial H)\right\}<\infty .
\end{aligned}
$$

Thus, by the compactness Corollary 2.12, there exists a subsequence $\left\{R_{i^{\prime}}\right\}$ which converges in the flat- $\alpha$ distance to some real flat chain, say $\widehat{R}$. By the definition of infimum and since the $\mathbf{M}^{\alpha}$ and $\mathbf{F i l l}{ }^{\alpha}$ masses are lower 
semicontinuous with respect to the flat- $\alpha$ norm, we find

$$
\begin{aligned}
L \leq G_{H}(\widehat{R}) & =\left[\mathbf{M}^{\alpha}(\widehat{R})\right]^{2}+\frac{1}{h}\left[\mathbf{F i l l}^{\alpha}(H-\widehat{R})\right]^{2}, \\
& \leq \liminf _{i^{\prime} \rightarrow \infty}\left[\mathbf{M}^{\alpha}\left(R_{i^{\prime}}\right)\right]^{2}+\liminf _{i^{\prime} \rightarrow \infty} \frac{1}{h}\left[\text { Fill }^{\alpha}\left(H-R_{i^{\prime}}\right)\right]^{2}, \\
& \leq \lim _{i^{\prime} \rightarrow \infty} G_{H}\left(R_{i^{\prime}}\right)=L .
\end{aligned}
$$

We also have

$$
\partial \widehat{R}=\lim _{i^{\prime} \rightarrow \infty} \partial R_{i^{\prime}}=\partial H
$$

where the limit is with respect to the flat- $\alpha$ norm.

Now, using $\widehat{R}$, define $R$ so that its support is contained in $K$. Let $f: \mathbb{R}^{m} \rightarrow K$ be the nearest point projection map so that

$$
|f(x)-x|=\inf \{|y-x|: y \in K\}
$$

Note that $f$ is a Lipschitz map with Lipschitz constant no greater than 1 . Set $R:=f_{\sharp} \widehat{R}$. By Lemma 2.19, Lemma 2.27, and the property that $f(x)=x$ for every $x \in K$, we know

$$
\begin{aligned}
G_{H}(R) & =\left[\mathbf{M}^{\alpha}(R)\right]^{2}+\frac{1}{h}\left[\mathbf{F i l l}^{\alpha}(H-R)\right]^{2}, \\
& \leq\left[\mathbf{M}^{\alpha}(\widehat{R})\right]^{2}+\frac{1}{h}\left[\operatorname{Fill}^{\alpha}(H-\widehat{R})\right]^{2}, \\
& \leq L .
\end{aligned}
$$

Moreover,

$$
\partial R=\partial f_{\sharp} \widehat{R}=f_{\sharp} \partial \widehat{R}=f_{\sharp} \partial H=\partial H .
$$

Corollary 3.3. For $R$ minimizing $G_{H}$ in the above proof, $\mathbf{M}^{\alpha}(R) \leq \mathbf{M}^{\alpha}(H)$.

Proof. This follows from defining $R$ in such a way that ensures

$$
\left[\mathbf{M}^{\alpha}(R)\right]^{2} \leq G_{H}(R)=\left[\mathbf{M}^{\alpha}(R)\right]^{2}+\frac{1}{h}\left[\mathbf{F i l l}^{\alpha}(H-R)\right]^{2} \leq G_{H}(H)=\left[\mathbf{M}^{\alpha}(H)\right]^{2} .
$$

We now inductively define the sequence of real-valued flat chains $\left\{T_{j}^{h}\right\}$ for the construction of the flow. Consider again $T_{0} \in \mathcal{N}_{k}^{\alpha}(K)$ having compact support. First, set $H=T_{0}^{h}=T_{0}$; and then, set $T_{1}^{h}:=\arg \min \left\{G_{H}(T)\right.$ : $T \in \mathcal{N}_{k}^{\alpha}(K)$ with compact support in $\left.K, \partial T=\partial T_{0}\right\}$, which we know exists by Theorem 3.2. Continue to inductively apply Theorem 3.2 to each $H=T_{j-1}^{h}$ to find $T_{j}^{h}$. By doing so, we have the following properties:

Lemma 3.4. The mass of the step minimizing sequence is non-increasing; i.e.

$$
\mathbf{M}^{\alpha}\left(T_{j}^{h}\right) \leq \mathbf{M}^{\alpha}\left(T_{j-1}^{h}\right) \leq \mathbf{M}^{\alpha}\left(T_{0}\right) .
$$




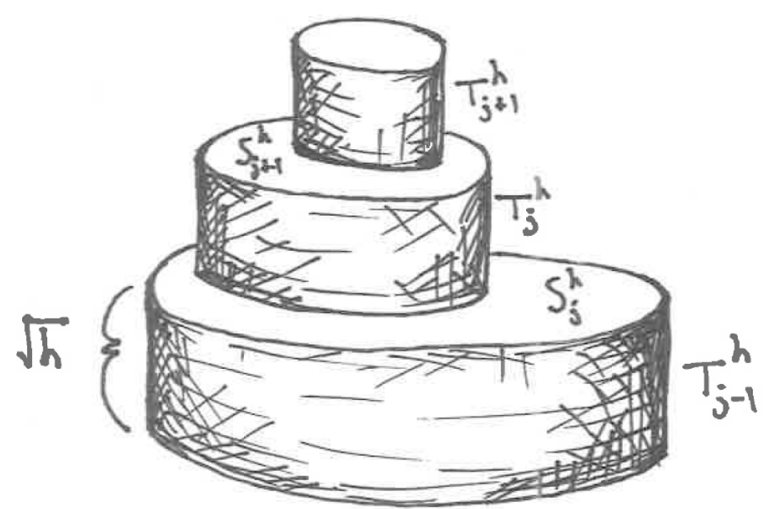

FiguRE 5. Shown here is a representation of a piece of the "birthday cake" decomposition of an $S_{h} \subset \mathbb{R}^{+} \times \mathbb{R}^{m}$, for $h>0$ defined in equation (3.3).

Additionally, we have the estimate

$$
\mathbf{F i l l}^{\alpha}\left(T_{j-1}^{h}-T_{j}^{h}\right) \leq \sqrt{h\left(\left[\mathbf{M}^{\alpha}\left(T_{j-1}^{h}\right)\right]^{2}-\left[\mathbf{M}^{\alpha}\left(T_{j}^{h}\right)\right]^{2}\right)} \leq \sqrt{h} \mathbf{M}^{\alpha}\left(T_{0}\right) .
$$

Proof. This lemma directly follows from the construction defined above. First Corollary 3.3 guarantees $\mathbf{M}^{\alpha}\left(T_{j}^{h}\right) \leq \mathbf{M}^{\alpha}\left(T_{j-1}^{h}\right)$. The second estimate results from rearranging the functional $G$ in equation (3.1) in the case where $T_{j}^{h}$ minimizes the functional for $H=T_{j-1}^{h}$ and using $G\left(T_{j}^{h}\right) \leq G\left(T_{j-1}^{h}\right)$.

\subsection{Construction of the flow}

To finish the construction of the flow, we will find a higher dimensional chain whose slices will dictate how to reduce the $\mathbf{M}^{\alpha}$ mass of the initial chain. Recall that we will do this by first creating the "birthday cake" decompositions $S_{h}$ out of each step minimizing sequence constructed above. The limit of these decompositions will result in our desired higher dimensional chain $S$ whose slices dictate the flow.

For every step $h>0$, we know from Lemma 3.4 that Fill $^{\alpha}\left(T_{j-1}^{h}-T_{j}^{h}\right)<\infty$. Note that $\partial\left(T_{j-1}^{h}-T_{j}^{h}\right)=0$ by construction. So, by Theorem 2.28 , there exists a $(k+1)$-dimensional real-valued flat chain $S_{j}^{h}$ satisfying $\partial S_{j}^{h}=T_{j-1}^{h}-T_{j}^{h}$ and

$$
\mathbf{M}^{\alpha}\left(S_{j}^{h}\right)=\mathbf{F i l l}^{\alpha}\left(T_{j-1}^{h}-T_{j}^{h}\right) \leq \sqrt{h} \mathbf{M}^{\alpha}\left(T_{0}\right)
$$

by the estimate found in Lemma 3.4.

Using a "birthday cake" decomposition, construct a $(k+1)$-dimensional, real-valued chain in $\mathbb{R}^{+} \times \mathbb{R}^{m}$ as follows. Let

$$
S_{h}:=\sum_{j=1}^{\infty}\left(-[|(j-1) \sqrt{h}, j \sqrt{h}|] \times T_{j}^{h}+[|(j-1) \sqrt{h}|] \times S_{j}^{h}\right) .
$$

See Figure 5 for a representation of such an $S_{h}$. 
Computing the boundary of $S_{h}$ with analogous techniques of Section 2.3, we find

$$
\begin{aligned}
\partial S_{h}= & \sum_{j=1}^{\infty} \partial\left(-[|(j-1) \sqrt{h}, j \sqrt{h}|] \times T_{j}^{h}+[|(j-1) \sqrt{h}|] \times S_{j}^{h}\right), \\
= & \sum_{j=1}^{\infty}\left([|(j-1) \sqrt{h}|] \times T_{j}^{h}-[|j \sqrt{h}|] \times T_{j}^{h}\right. \\
& \left.\quad+[|(j-1) \sqrt{h}, j \sqrt{h}|] \times \partial T_{j}^{h}+[|(j-1) \sqrt{h}|] \times \partial S_{j}^{h}\right), \\
= & \sum_{j=1}^{\infty}\left([|(j-1) \sqrt{h}|] \times T_{j}^{h}-[|j \sqrt{h}|] \times T_{j}^{h}\right. \\
& \left.\quad+[|(j-1) \sqrt{h}, j \sqrt{h}|] \times \partial T_{0}+[|(j-1) \sqrt{h}|] \times\left(T_{j-1}^{h}-T_{j}^{h}\right)\right), \\
= & \sum_{j=1}^{\infty}\left([|(j-1) \sqrt{h}|] \times T_{j-1}^{h}-[|j \sqrt{h}|] \times T_{j}^{h}\right)+\sum_{j=1}^{\infty}\left([|(j-1) \sqrt{h}, j \sqrt{h}|] \times \partial T_{0}\right), \\
= & {[|0|] \times T_{0}-\lim _{j \rightarrow \infty}[|j \sqrt{h}|] \times T_{j}^{h}+[|0,+\infty|] \times \partial T_{0} . }
\end{aligned}
$$

Ultimately, we wish to take a limit of these $S_{h}$ as $h \rightarrow 0$, but in order for such a limit to converge, we must restrict $S_{h}$ to some finite time interval cross $\mathbb{R}^{m}$ so that the sequence of these higher dimensional chains has a bounded supremum of $\mathbf{M}^{\alpha}$ mass and $\mathbf{M}^{\alpha}$ boundary mass.

Lemma 3.5. Given $C \in \mathbb{R}^{+}$and fixed $h>0$, then the $\mathbf{M}^{\alpha}$ mass and $\mathbf{M}^{\alpha}$ boundary mass of the restriction $S_{h}\left\llcorner[0, C] \times \mathbb{R}^{m}\right.$ is bounded and

$$
\mathbf{N}^{\alpha}\left(S_{h}\left\llcorner[0, C] \times \mathbb{R}^{m}\right) \leq(2 C+2) \mathbf{M}^{\alpha}\left(T_{0}\right)+C \mathbf{M}^{\alpha}\left(\partial T_{0}\right) .\right.
$$

Proof. Given $C>0$ and $h>0$, choose integer $\ell$ so that $(\ell-1) \sqrt{h}<C \leq \ell \sqrt{h}$. Using the triangle inequality, equation (2.5), Lemma 3.4, and Inequality 3.2, compute

$$
\begin{aligned}
\mathbf{M}^{\alpha}\left(S_{h}\left\llcorner[0, C] \times \mathbb{R}^{m}\right)\right. & \leq \sum_{j=1}^{\ell}\left(\mathbf{M}^{\alpha}\left(-[|(j-1) \sqrt{h}, j \sqrt{h}|] \times T_{j}^{h}\right)+\mathbf{M}^{\alpha}\left([|(j-1) \sqrt{h}|] \times S_{j}^{h}\right)\right), \\
& \leq \sum_{j=1}^{l}\left(\sqrt{h} \mathbf{M}^{\alpha}\left(T_{j}^{h}\right)+\mathbf{M}^{\alpha}\left(S_{j}^{h}\right)\right), \\
& \leq \sum_{j=1}^{l}\left(\sqrt{h} \mathbf{M}^{\alpha}\left(T_{0}\right)+\sqrt{h} \mathbf{M}^{\alpha}\left(T_{0}\right)\right), \\
& =(\ell-1)\left(2 \sqrt{h} \mathbf{M}^{\alpha}\left(T_{0}\right)\right), \\
& <2 C \mathbf{M}^{\alpha}\left(T_{0}\right) .
\end{aligned}
$$

Computing the boundary of this restricted chain, one can show that

$$
\partial\left(S_{h}\left\llcorner[0, C] \times \mathbb{R}^{m}\right)=[|0|] \times T_{0}-[|C|] \times T_{\ell}^{h}+[|0, C|] \times \partial T_{0} .\right.
$$


So, using equation (2.5) and Lemma 3.4, we find

$$
\begin{aligned}
\mathbf{M}^{\alpha}\left(\partial\left(S_{h}\left\llcorner[0, C] \times \mathbb{R}^{m}\right)\right)\right. & \leq \mathbf{M}^{\alpha}\left(T_{0}\right)+\mathbf{M}^{\alpha}\left(T_{\ell}^{h}\right)+C \mathbf{M}^{\alpha}\left(\partial T_{0}\right), \\
& \leq 2 \mathbf{M}^{\alpha}\left(T_{0}\right)+C \mathbf{M}^{\alpha}\left(\partial T_{0}\right) .
\end{aligned}
$$

Combining these bounds, we find

$$
\mathbf{N}^{\alpha}\left(S_{h}\left\llcorner[0, C] \times \mathbb{R}^{m}\right) \leq(2 C+2) \mathbf{M}^{\alpha}\left(T_{0}\right)+C \mathbf{M}^{\alpha}\left(\partial T_{0}\right) .\right.
$$

Using this lemma, we will now find our limiting chain $S$ whose slices will dictate the flow.

Theorem 3.6. Given $C \in \mathbb{R}^{+}$. Let $S_{i}$ be an abbreviation for the real flat chain $S_{h}\left\llcorner[0, C] \times \mathbb{R}^{m}\right.$ with $h=2^{-i}$. There exists a subsequence of $\left\{S_{i}\right\}$ converging to a real flat chain $S$ with $\partial S=[|0|] \times T_{0}-[|C|] \times T_{C}+[|0, C|] \times$ $\partial T_{0}$, where $T_{C} \in \mathcal{N}_{k}^{\alpha}(K)$ with $\partial T_{C}=\partial T_{0}$.

Proof. Applying Lemma 3.5 to $h=2^{-i}$, we find

$$
\sup _{i}\left\{\mathbf{N}^{\alpha}\left(S_{i}\right)\right\} \leq(2 C+2) \mathbf{M}^{\alpha}\left(T_{0}\right)+C \mathbf{M}^{\alpha}\left(\partial T_{0}\right)<\infty .
$$

By the Compactness Corollary 2.12, there exists a subsequence $\left\{S_{i^{\prime}}\right\}$ converging to some real flat chain $S$ in the flat- $\alpha$ norm.

Since $S_{i^{\prime}}$ converges to $S$ in the flat- $\alpha$ norm, $\partial S_{i^{\prime}} \rightarrow \partial S$ in the flat- $\alpha$ norm. In the proof of Lemma 3.5, we computed for each $h=2^{-i^{\prime}}$,

$$
\partial\left(S_{h}\left\llcorner[0, C] \times \mathbb{R}^{m}\right)=[|0|] \times T_{0}-[|C|] \times T_{\ell}^{h}+[|0, C|] \times \partial T_{0},\right.
$$

where $\ell \in \mathbb{Z}$ depends on $h$ and $(\ell-1) \sqrt{h}<C \leq \ell \sqrt{h}$. As $h \rightarrow 0, \ell \sqrt{h} \rightarrow C$. Since $\left\{\partial S_{i^{\prime}}\right\}$ converges with respect to the flat- $\alpha$ norm, we have $\left\{T_{\ell}^{h}\right\}$ for $h=2^{-i^{\prime}}$ converge with respect to the flat- $\alpha$ norm to some real flat chain $T_{C} \in \mathcal{N}_{k}^{\alpha}(K)$ with $\partial T_{C}=\partial T_{0}$.

See Figure 6 for a visual representation of $S$.

Now, we will define the slices of $S$ that will dictate the flow and show that these slices have the desired properties: mass decreasing, boundary preserving, and Lipschitz continuous with respect to the flat- $\alpha$ norm.

Let $p: \mathbb{R}^{+} \times \mathbb{R}^{m} \rightarrow \mathbb{R}^{+}$be the projection defined by $p(t, x)=t$. Denote the slice of $S$ by $p$ at $t>0$ by $\left\langle S, p, t_{+}\right\rangle$. Note that this slice is a finite $\mathbf{M}^{\alpha}$ mass, $k$-dimensional flat chain for almost every $t>0$. Similarly, we may define the slices of $S_{i}$ for each $i$ and again have $\left\langle S_{i}, p, t_{+}\right\rangle \in \mathcal{N}_{k}^{\alpha}([|0, C|] \times K)$. See Figure 7 for a representation of these slices.

Set

$$
T_{t}:=\pi_{\sharp}\left\langle S, p, t_{+}\right\rangle
$$

where $\pi: \mathbb{R} \times \mathbb{R}^{m} \rightarrow \mathbb{R}^{m}$ is the projection map defined by $\pi(t, x)=x$. We will utilize the slicing results, Lemma 2.31 and Corollary 2.32, to prove the following theorems:

Theorem 3.7. Given $C \in \mathbb{R}^{+}$. For almost every $t$ such that $0 \leq t \leq C$,

$$
\mathbf{M}^{\alpha}\left(T_{t}\right) \leq \mathbf{M}^{\alpha}\left(T_{0}\right)
$$




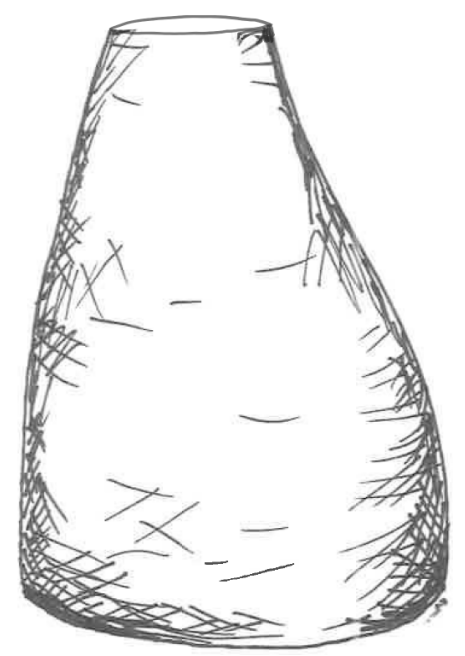

FIgURE 6 . Shown here is a piece of the $(k+1)$-dimensional chain $S \subset \mathbb{R}^{+} \times \mathbb{R}^{m}$; the slices of suitable restrictions dictate the flow.
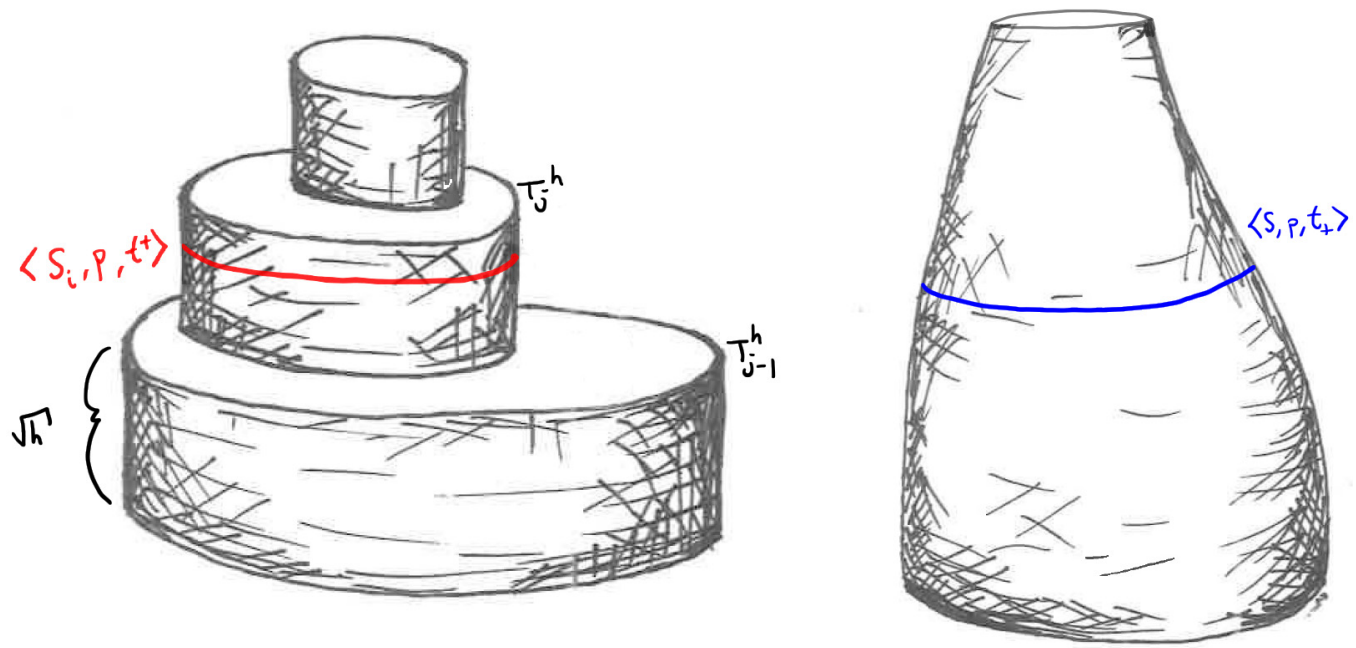

FIGURE 7 . Shown here is a representation of $\left\langle S_{i}, p, t_{+}\right\rangle$on the left and $\left\langle S, p, t_{+}\right\rangle$on the right.

and

$$
\partial T_{t}=\partial T_{0}
$$

Proof. Note that Lemma 3.4 already shows this result for when $t$ is a dyadic rational number. Otherwise, by equation (3.3),

$$
\left\langle S_{i}, p, t_{+}\right\rangle=[|t|] \times T_{j}^{h},
$$

where $h=2^{-i}$ and $j$ is the largest integer such that $(j-1) \sqrt{h}<t \leq j \sqrt{h}$. (See Fig. 7.) By the Slicing Lemma 2.31, we know that for almost every $t>0$, there exists a subsequence $i^{\prime}$ in which the convergence 
is "quick," that is,

$$
\sum_{j^{\prime}=1}^{\infty} \mathbf{F}^{\alpha}\left(\left\langle S_{i^{\prime}}-S, p, t_{+}\right\rangle\right)<\infty
$$

so that

$$
\mathbf{F}^{\alpha}\left(\left\langle S_{i^{\prime}}, p, t_{+}\right\rangle-\left\langle S, p, t_{+}\right\rangle\right) \rightarrow 0
$$

Thus, for all such $t$ that are not dyadic rationals, we have for $h^{\prime}=2^{-i^{\prime}}$

$$
\begin{aligned}
\mathbf{F}^{\alpha}\left(\pi_{\sharp}\left(\left\langle S_{i^{\prime}}, p, t_{+}\right\rangle\right)-\pi_{\sharp}\left(\left\langle S, p, t_{+}\right\rangle\right)\right) & \rightarrow 0 ; \\
\mathbf{F}^{\alpha}\left(\pi_{\sharp}\left([|t|] \times T_{j}^{h^{\prime}}\right)-T_{t}\right) & \rightarrow 0 ; \\
\mathbf{F}^{\alpha}\left(T_{j}^{h^{\prime}}-T_{t}\right) & \rightarrow 0 .
\end{aligned}
$$

By the lower semicontinuity of $\mathbf{M}^{\alpha}$ mass of slices with respect to the flat- $\alpha$ distance (Cor. 2.32) and by Lemma 3.4,

$$
\mathbf{M}^{\alpha}\left(T_{t}\right) \leq \liminf _{i^{\prime} \rightarrow \infty} \mathbf{M}^{\alpha}\left(T_{j}^{h^{\prime}}\right) \leq \mathbf{M}^{\alpha}\left(T_{0}\right)
$$

Moreover, by construction, we have for almost every $t \in[0, C]$

$$
\partial T_{t}=\lim _{i^{\prime} \rightarrow \infty} \partial T_{j}^{h^{\prime}}=\partial T_{0}
$$

where $h^{\prime}=2^{-i^{\prime}}$ and the limit is with respect to the flat- $\alpha$ norm.

Of course, the interesting examples are when the inequality in Theorem 3.7 is strict, which happens when $T_{0}$ is not a local minimum of the functional $G$ defined in equation (3.1). We will explore multiple such non-trivial examples in which mass actually decreases in the following section. But first, we will complete the proof of Theorem 3.1 by proving the remaining Lipschitz continuous property:

Theorem 3.8. Let $C \in \mathbb{R}^{+}$. The constructed flow is Lipschitz continuous in time with respect to the flat- $\alpha$ norm; i.e., for $0 \leq t<r \leq C$,

$$
\mathbf{F}^{\alpha}\left(T_{r}-T_{t}\right) \leq(r-t) \mathbf{M}^{\alpha}\left(T_{0}\right)
$$

Proof. Let $0 \leq t<r \leq C$. Since $\partial S=[|0|] \times T_{0}-[|C|] \times T_{C}+[|0, C|] \times \partial T_{0}$, where $T_{C} \in \mathcal{N}_{k}^{\alpha}(K)$ with $\partial T_{C}=\partial T_{0}$,

$$
\begin{aligned}
\left\langle S, p, r_{+}\right\rangle-\left\langle S, p, t_{+}\right\rangle & =\partial\left(S\left\llcorner\left\{(t, r] \times \mathbb{R}^{m}\right\}\right)-(\partial S)\left\llcorner\left\{(t, r] \times \mathbb{R}^{m}\right\},\right.\right. \\
& =\partial\left(S\left\llcorner\left\{(t, r] \times \mathbb{R}^{m}\right\}\right)-[|t, r|] \times \partial T_{0} .\right.
\end{aligned}
$$

Thus,

$$
\begin{aligned}
T_{r}-T_{t} & =\pi_{\sharp}\left(\partial\left(S\left\llcorner\left\{(t, r] \times \mathbb{R}^{m}\right\}\right)-[|t, r|] \times \partial T_{0}\right),\right. \\
& =\partial \pi_{\sharp}\left(S\left\llcorner\left\{(t, r] \times \mathbb{R}^{m}\right\}\right) .\right.
\end{aligned}
$$


Since the flat- $\alpha$ norm is lower semicontinuous, then for $\left\{i^{\prime}\right\}$ as in the proof for Theorem 3.7, we have

$$
\begin{aligned}
\mathbf{F}^{\alpha}\left(T_{r}-T_{t}\right) & \leq \mathbf{F}^{\alpha}\left(\partial \pi_{\sharp}\left(S\left\llcorner\left\{(t, r] \times \mathbb{R}^{m}\right\}\right)\right),\right. \\
& \leq \liminf _{i^{\prime} \rightarrow \infty} \mathbf{F}^{\alpha}\left(\partial \pi_{\sharp}\left(S_{i^{\prime}}\left\llcorner\left\{(t, r] \times \mathbb{R}^{m}\right\}\right)\right),\right. \\
& \leq \liminf _{i^{\prime} \rightarrow \infty} \mathbf{M}_{k+1}^{\alpha}\left(\pi_{\sharp}\left(S_{i^{\prime}}\left\llcorner\left\{(t, r] \times \mathbb{R}^{m}\right\}\right)\right),\right. \\
& \leq(r-t) \mathbf{M}^{\alpha}\left(T_{0}\right) .
\end{aligned}
$$

To see the last inequality, choose $\ell, \tilde{\ell}$ so that

$$
(\ell-1) \sqrt{h^{\prime}}<t \leq \ell \sqrt{h^{\prime}} \text { and }(\tilde{\ell}-1) \sqrt{h^{\prime}}<r \leq \tilde{\ell} \sqrt{h^{\prime}},
$$

where $h^{\prime}=2^{-i^{\prime}}$. Then,

$$
\begin{aligned}
\mathbf{M}_{k+1}^{\alpha}\left(\pi_{\sharp}\left(S_{i^{\prime}}\left\llcorner\left\{(t, r] \times \mathbb{R}^{m}\right\}\right)\right)\right. & \leq \mathbf{M}_{k+1}^{\alpha}\left(\pi_{\sharp}\left(\sum_{j=\ell}^{\tilde{\ell}}-\left[\left|(j-1) \sqrt{h^{\prime}}, j \sqrt{h^{\prime}}\right|\right] \times T_{j}^{h^{\prime}}+\left[\left|(j-1) \sqrt{h^{\prime}}\right|\right] \times S_{j}^{h^{\prime}}\right)\right), \\
& =\mathbf{M}_{k+1}^{\alpha}\left(\sum_{j=\ell}^{\tilde{\ell}} S_{j}^{h^{\prime}}\right),
\end{aligned}
$$

where this last equality follows from the definition of projection $\pi$ and the fact that $\mathcal{H}^{k+1}\left(T_{j}^{h^{\prime}}\right)=0$ (since each $T_{j}^{h^{\prime}}$ is $k$-dimensional). Finally, using the triangle inequality, Inequality (3.2), and our choice of $\ell, \tilde{\ell}$, we find that the above is bounded by

$$
\begin{aligned}
\mathbf{M}_{k+1}^{\alpha}\left(\sum_{j=\ell}^{\tilde{\ell}} S_{j}^{h^{\prime}}\right) & \leq \sum_{j=\ell}^{\tilde{\ell}} \mathbf{M}^{\alpha}\left(S_{j}^{h^{\prime}}\right), \\
& \leq \sum_{j=\ell}^{\tilde{\ell}} \sqrt{h^{\prime}} \mathbf{M}^{\alpha}\left(T_{0}\right), \\
& \leq\left(r-t+\sqrt{h^{\prime}}\right) \mathbf{M}^{\alpha}\left(T_{0}\right),
\end{aligned}
$$

finishing the proof.

This concludes the proof of the paper's main Theorem 3.1, but we remind the reader here of its outline as well as the intuitive analogy of flip books. Recall that in Section 3.1, we first found step minimizing sequences of chains, which we conceptualized as the pages of flip books. Out of these sequences, we created "birthday cake" decompositions, $S_{h}$ 's, at the beginning of Section 3.2, and we conceptualized these as sewing together those pages into flip books. Finally, we found real flat chain $S$ in Theorem 3.6 through a convergence argument, and we imagined $S$ to be the animation obtained from the limit of the flip books as each page changes less and less from the previous page $(h \rightarrow 0)$. The "time slices" $T_{t}$ of $S$ are the chains in Theorem 3.1, and Theorems 3.7 and 3.8 showed that the flow dictated by these $T_{t}$ indeed possess the properties claimed in Theorem 3.1: the flow is mass decreasing, boundary preserving, and Lipschitz continuous with respect to the flat- $\alpha$ norm.

For transport networks, as explored in Section 4, the "flip book-like" constructed flow would appear to reduce $\mathbf{M}^{\alpha}$ cost through a "(un)zipping" process while not altering the boundary. For example, time stamps of this process on a particular transport path may be seen in Figure 1; in a sense, these time stamps could be imagined to be pages of such a flip book animation described above. 


\section{Applications to transportation networks}

We wish to investigate the structure of optimal branched transport paths that accommodate efficiencies of scale into the model, and thus, we will consider such paths as real-valued, 1-dimensional flat chains with an $\mathbf{M}^{\alpha}$ cost function. Instead of solving this optimization problem outright, we will apply our constructed cost reducing flow to evolve transport networks. We will see in examples how this minimizing flow encourages overlapping structures because of the $\mathbf{M}^{\alpha}$ mass term in functional $G$ in equation (3.1). Thus, the functional $G$ is particularly amenable to evolving branched transport networks in cost reducing ways through a "(un)-zipping" process, creating or eliminating interior vertices. In fact, the ability of our geometric flow to alter the network's underlying topology is particularly advantageous since many of the computational complications of current numerical methods stem from not a priori knowing the topology of the optimal network. It is our hope that the constructed flow, especially the discrete step minimizing process, helps further research on computational methods of optimizing branched transport networks.

Since the underlying theme of geometric flows is to gain insight into difficult optimization problems through indirect methods, minimizing flows are only able to find local optimal solutions. Essentially, minimizing flows are only able to describe how to reduce the functional of the currently considered object and are unable to foresee if first increasing and then decreasing that functional would actually lead to a better solution. Our constructed flow is no different and will not guarantee convergence to the optimal solution but instead evolve an initial network to a locally optimal branched network. However, this "nearest" locally optimal solution might be significantly geometrically and topologically "far away" from the initial path.

In this section, we will first give background on the branched transport problem and then analyze a few examples showing how the flow evolves networks. The example developed in Section 4.2.1 shows an initial path flowing to the global optimal solution. In Section 4.2.2, we will see an example merely flowing to a local minimum to show that indeed our minimizing flow is no different than others in this regard. However, we will also see in that subsection how certain shapes of initial transport networks appear to always flow to the global optimal solution even in strikingly different boundary configurations.

\subsection{Preliminaries for transportation networks}

Let $\mu^{+}, \mu^{-}$be two atomic measures of equal mass supported in closed and convex $K \subset \mathbb{R}^{m}$; say

$$
\mu^{+}=\sum_{i=1}^{k} m_{i} \delta_{w_{i}} \text { and } \mu^{-}=\sum_{j=1}^{\ell} n_{j} \delta_{v_{j}}
$$

where $\sum_{i=1}^{k} m_{i}=\sum_{j=1}^{\ell} n_{j}$. The $\left\{w_{i}\right\}_{i=1}^{k}$ are called sources and the $\left\{v_{j}\right\}_{j=1}^{\ell}$ are called sinks.

Definition 4.1. A transportation network, or transport path, from $\mu^{+}$to $\mu^{-}$is a weighted directed graph $P$ whose vertex set $V(P)$, directed edge set $E(P)$, and weight function $\lambda: E(P) \rightarrow(0,+\infty)$ satisfy $\left\{w_{i}\right\}_{i=1}^{k} \cup$ $\left\{v_{j}\right\}_{j=1}^{\ell} \subset V(P)$ and for any vertex $x \in V(P)$,

$$
\sum_{e \in E(P), e^{-}=x} \lambda(e)=\sum_{e \in E(P), e^{+}=x} \lambda(e)+ \begin{cases}m_{i} & \text { if } x=w_{i} \text { for some } i=1, \ldots, k \\ -n_{j} & \text { if } x=v_{j} \text { for some } j=1, \ldots, \ell \\ 0 & \text { otherwise }\end{cases}
$$

where $e^{-}$and $e^{+}$denote the starting and ending endpoints of edge $e \in E(P)$, respectively. 
Let $\operatorname{Path}\left(\mu^{+}, \mu^{-}\right)$denote the space of all transport paths from $\mu^{+}$to $\mu^{-}$, which is always nonempty because the following "coned" network satisfies the above conditions:

$$
P=\sum_{i=1}^{k} m_{i}\left[\left|\left(w_{i}, x\right)\right|\right]+\sum_{j=1}^{\ell} n_{j}\left[\left|\left(x, v_{j}\right)\right|\right],
$$

where $x \in K$. Note that one may consider a transport path $P$ from $\mu^{+}$to $\mu^{-}$as a 1-dimensional, real-valued polyhedral chain as in Definition 2.1. Thus, $\operatorname{Path}\left(\mu^{+}, \mu^{-}\right) \subset \mathcal{P}_{1}(K)$. The balance equation (4.1) can be thought of as Kirchhoff's law or as mass being conserved at each vertex. In terms of polyhedral chains, this means that $\partial P=\mu^{-}-\mu^{+}$when one views $\mu^{+}, \mu^{-}$as 0 -dimensional chains.

Definition 4.2. For any $\alpha \in[0,1]$ and for any $P \in \operatorname{Path}\left(\mu^{+}, \mu^{-}\right)$, we may define the transportation cost of $P$ to be its $\mathbf{M}^{\alpha}$ mass:

$$
\mathbf{M}^{\alpha}(P):=\sum_{e \in E(P)}[\lambda(e)]^{\alpha} \mathcal{H}^{1}(e) .
$$

Lemma 4.3. [41] For any polyhedral 1-dimensional chain $P$, there exists a polyhedral 1-dimensional chain $\tilde{P}$ containing no cycles in its support with $\partial P=\partial \tilde{P}$ and $\mathbf{M}^{\alpha}(\tilde{P}) \leq \mathbf{M}^{\alpha}(P)$.

For continuous extensions of Gilbert's model, see [5, 27, 41]. However, since our flow requires the initial chain to have finite boundary mass, we only focus on transportation networks between finite atomic measures. Additionally, we may restrict our attention in this section to transport paths as polyhedral chains because we may approximate any flat chain by a polyhedral chain in the flat- $\alpha$ norm. In fact, we will further restrict our attention to only polyhedral chains without cycles. Note that the constructed flow does not guarantee that every chain $T_{t}$ from the flow has a tree structure, and it may indeed be advantageous in functional $G$ in equation (3.1) to sacrifice an increase in the $\mathbf{M}^{\alpha}$ mass term to carve out some area using a cycle to decrease the fill- $\alpha$ term. To simplify investigating the flow on paths using analytic methods, we will restrict our attention to polyhedral chains without cycles resulting still in flowing the initial chain to local minima due to Lemma 4.3.

Note that since the flow required $\alpha \in(0,1]$ for the convergence arguments to hold, we will only be able to investigate reducing the $\mathbf{M}^{\alpha}$ cost for these $\alpha$ values. Thus, our flow will not apply to finding solutions to the case where $\alpha=0$, famously known as Steiner trees.

\subsection{Examples}

When applying the flow to some initial transportation network, one transforms the network, possibly changing the topology of the initial path through a "(un)zipping" process, creating or eliminating bifurcation points in cost reducing ways. As discussed above, we will restrict our attention here to only polyhedral chain transport paths without cycles. We will analyze examples where the supply and demand distributions are two sources/one sink and two sources/two sinks, as the optimal paths are known in these cases.

\subsubsection{Two sources; one sink}

Let $\alpha \in[0,1], \mu^{+}=m_{1} \delta_{w_{1}}+m_{2} \delta_{w_{2}}$, and $\mu^{-}=\left(m_{1}+m_{2}\right) \delta_{v}$, where $m_{1}, m_{2} \in \mathbb{R}$ and $w_{1}, w_{2}, v \in \mathbb{R}^{2}$ are distinct. In this case, we know the optimal transportation network has either a "V" shape, an "L" shape, or a "Y" shape [6, 41]; for the "Y" shaped case, we further know the location of the optimal branching point as given in the lemma below. 


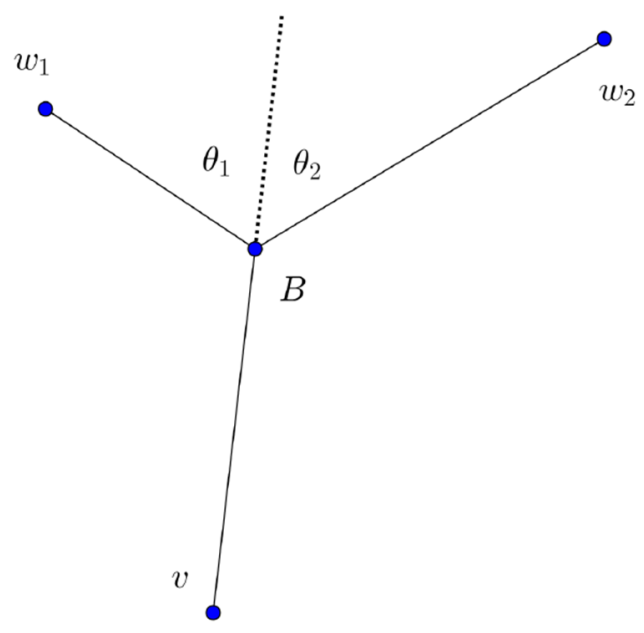

Figure 8 . If $P$ is optimal and has three edges as shown, then $\theta_{1}$ and $\theta_{2}$ must satisfy the angle constraints in Lemma 4.4 .

Lemma 4.4. [41] If $P \in P$ Path $\left(\mu^{+}, \mu^{-}\right)$is optimal and made up of three edges, then the interior vertex $B$ of $P$ must satisfy the following angle constraints:

$$
\begin{aligned}
& \cos \left(\theta_{1}\right)=\frac{k_{1}^{2 \alpha}+1-k_{2}^{2 \alpha}}{2 k_{1}^{\alpha}} ; \\
& \cos \left(\theta_{2}\right)=\frac{k_{2}^{2 \alpha}+1-k_{1}^{2 \alpha}}{2 k_{2}^{\alpha}} ;
\end{aligned}
$$

and

$$
\cos \left(\theta_{1}+\theta_{2}\right)=\frac{1-k_{1}^{2 \alpha}-k_{2}^{2 \alpha}}{2 k_{1}^{\alpha} k_{2}^{\alpha}},
$$

where $k_{1}=\frac{m_{1}}{m_{1}+m_{2}}, k_{2}=\frac{m_{2}}{m_{1}+m_{2}}$, and $\theta_{1}, \theta_{2}$ are as shown in Figure 8 .

We will examine how the flow evolves an initial path having a "V" shape to the optimal transport path.

Again, Let $\mu^{+}=m_{1} \delta_{w_{1}}+m_{2} \delta_{w_{2}}$ and $\mu^{-}=\left(m_{1}+m_{2}\right) \delta_{v}$, where $m_{1}, m_{2} \in \mathbb{R}$ and $w_{1}, w_{2}, v \in \mathbb{R}^{2}$ are distinct, but this time let $\alpha \in(0,1]$. Let's additionally assume that the optimal transport network has a "Y" shape. Without loss of generality, set $v=(0,0)$ and let the positive $x$-axis extend from $v$ to $B=\left(x_{B}, 0\right)$ where $B$ is the optimal bifurcation point for the "Y" shape; that is, the $x$-axis breaks the angle $\angle w_{1} B w_{2}$ into $\theta_{1}$ and $\theta_{2}$ in Lemma 4.4.

Let us see how the flow zips up the initial path $T_{0} \in \operatorname{Path}\left(\mu^{+}, \mu^{-}\right)$having the "V" structure; i.e.,

$$
T_{0}=m_{1}\left[\left|w_{1}, v\right|\right]+m_{2}\left[\left|w_{2}, v\right|\right] .
$$

Lemma 4.5. One may choose each $T_{j}^{h}$ for every $h>0$ in the discrete step minimizing process to be a " $Y$ " shaped path with bifurcation point on the $x$-axis, and thus, ensure that each $T_{t}$ for almost every $t>0$ has this same "Y" shaped structure. 


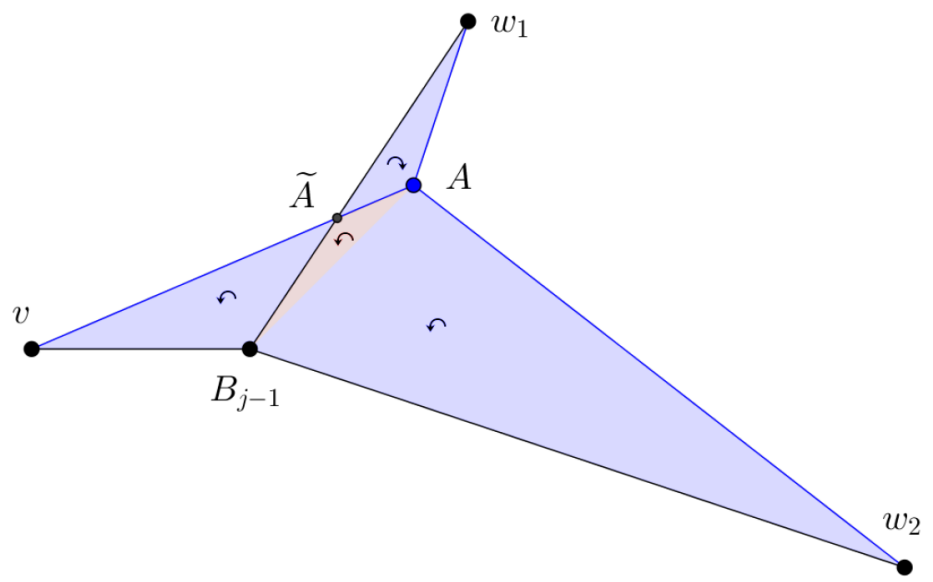

FiguRE 9. Inductively showing each minimizer is "Y" shaped in Lemma 4.5.

Proof. We may assume that each path in the discrete sequences is contained in the convex hull of $w_{1}, w_{2}$, and $v[5]$.

Now fix $h>0$. We will prove via induction that every polyhedral path in our discrete sequence is "Y" shaped and its interior vertex lies on the $x$-axis. Let

$$
T_{j-1}^{h}=m_{1}\left[\left|w_{1}, B_{j-1}\right|\right]+m_{2}\left[\left|w_{2}, B_{j-1}\right|\right]+\left(m_{1}+m_{2}\right)\left[\left|B_{j-1}, v\right|\right]
$$

where $B_{j-1}=\left(x_{j-1}, 0\right)$. (For $j=1, B_{j-1}=(0,0)$ so that $T_{j-1}^{h}$ is exactly $T_{0}$.)

Suppose $T$ minimizes functional $G$ in equation (3.1) (for $H=T_{j-1}^{h}$ ), and let $A=(x, y)$ be its interior vertex. (For the existence of such an $A$, we are relying on our restriction to polyhedral chains without cycles.) By Lemma 2.19, we may use projection to decrease the $\mathbf{M}^{\alpha}$ mass (and also, the $\mathbf{F i l l}{ }^{\alpha}$ norm), and so, we may assume that $A$ lies in the convex hull of $v, w_{1}$ and $w_{2}$. Without loss of generality, assume that the edge $[|A, v|]$ intersects $\left[\left|w_{1}, B_{j-1}\right|\right]$ at $\widetilde{A}=(\tilde{x}, \tilde{y})$. See Figure 9 .

Then, we have

$$
\begin{aligned}
G(T) & =\left[\mathbf{M}^{\alpha}(T)\right]^{2}+\frac{\left[\mathbf{F i l l}^{\alpha}\left(T-T_{j}^{h}\right)\right]^{2}}{h} \\
G(A) & =\left[m_{1}^{\alpha}\left\|\overrightarrow{A w_{1}}\right\|+m_{2}^{\alpha}\left\|\overrightarrow{A w_{2}}\right\|+\left(m_{1}+m_{2}\right)^{\alpha}\|\vec{A}\|\right]^{2} \\
& +\frac{1}{h}\left[\frac{m_{1}^{\alpha}}{2}\left\|\overrightarrow{\widetilde{A} w_{1}} \times \overrightarrow{A w_{1}}\right\|+\frac{m_{2}^{\alpha}}{2}\left\|\overrightarrow{A w_{2}} \times \overrightarrow{B_{j-1} w_{2}}\right\|+\frac{m_{2}^{\alpha}}{2}\left\|\overrightarrow{\widetilde{A} B_{j-1}} \times \overrightarrow{\widetilde{A} B_{j-1}}\right\|+\frac{\left(m_{1}+m_{2}\right)^{\alpha}}{2}\left\|\overrightarrow{B_{j-1}} \times \overrightarrow{\widetilde{A}}\right\|\right]^{2} .
\end{aligned}
$$

Writing the above equation in terms of $(x, y)$ (including expressing $(\tilde{x}, \tilde{y})$ in terms of $(x, y)$ ), one can show that the above function is convex in $y$. And so, we have

$$
G((x, 0)) \leq \frac{G(x, y)+G(x,-y)}{2}=G((x, y))
$$

Hence, there is a minimizer of $G$ that is "Y" shaped and its interior vertex lies on the $x$-axis, and we may choose this minimizer as $T_{j}^{h}$ in the flow's construction. 
Moreover, the flow will stop zipping once the path has reached the optimal shape. One may see this by examining how any perturbation of the network with the optimal bifurcation point will increase the functional in equation (3.1).

Remark 4.6. In fact, using that perturbation argument, one could show that if the initial "V" shaped network is the optimal one, the flow will keep this network fixed. And similar to the argument in Lemma 4.5, one may show that the flow unzips initial "Y" shaped paths with angles greater than that in Lemma 4.4 until the optimal bifurcation point is reached (or completely eliminating the bifurcation point if the "V" network is optimal). Furthermore, one may show that the flow zips up a "V" shaped initial path to the "L" shaped optimal network using the same technique. In this case, one would see a creation and subsequent elimination of an interior vertex in the networks as they flow from the "V" shape to the "L" shape. Thus, the flow always evolves the initial "V" shaped network to the minimum transportation network.

Remark 4.7. The language of "may choose" in Lemma 4.5 stresses the non-uniqueness of the flow due to perhaps having choices in the creation of the step minimizing sequence. This particularly happens with high levels of symmetry. For example, there are multiple global optimal networks connecting the source and sinks in Figure 1. (Think about rotations.) To flow to a different global minimum, one just needs to pick the $T_{1}^{h}$ in each step minimizing sequence that corresponds to this global minimum. (These choices in chains should just be rotations of each other.)

One may also compute the rate of zipping in specific examples:

Example 4.8. Let $\mu^{+}=\delta_{(0,1)}+\delta_{(0,-1)}$ and $\mu^{-}=2 \delta_{(2,0)}$, and let $\alpha=0.5$. Suppose the initial path is $T_{0}=$ $[|(0,1),(2,0)|]+[|(0,-1),(2,0)|]$. By Lemma 4.5 , we know the flow zips up the path along the $x$-axis and ceases once an angle of $\pi / 2$ is achieved. Here, we compute the rate of zipping.

Fix $h>0$. Suppose $\left(x_{j-1}, 0\right)$ is the bifurcation point of $T_{j-1}^{h}$. If $\left(x_{j}, 0\right)$ denotes the bifurcation point of $T_{j}^{h}$, then it must be a critical point of the functional:

$$
\begin{gathered}
G_{j}(x)=\left[\sqrt{2}(2-x)+2 \sqrt{x^{2}+1}\right]^{2}+\frac{\left[2 \frac{\left(x_{j-1}-x\right)}{2}\right]^{2}}{h} ; \\
0=G_{j}^{\prime}\left(x_{j}\right)=2\left[\sqrt{2}\left(2-x_{j}\right)+2 \sqrt{x_{j}^{2}+1}\right]\left[-\sqrt{2}+\frac{2 x_{j}}{\sqrt{x_{j}^{2}+1}}\right]-\frac{2\left(x_{j-1}-x_{j}\right)}{h} .
\end{gathered}
$$

To find the bifurcation point $(x(t), 0)$ then of $T_{t}$ for $t>0$, choose $\ell$ so that $\ell h \leq t<(\ell+1) h$ for each $h>0$. Rearranging equation (4.5) and summing, we have

$$
\sum_{j=1}^{\ell} x_{j}-x_{j-1}=-\sum_{j=1}^{\ell} h\left[\sqrt{2}\left(2-x_{j}\right)+2 \sqrt{x_{j}^{2}+1}\right]\left[-\sqrt{2}+\frac{2 x_{j}}{\sqrt{x_{j}^{2}+1}}\right]
$$

then, let $h \rightarrow 0$ :

$$
x(t)-x(0)=-\int_{0}^{t}\left[\sqrt{2}\left(2-x_{j}\right)+2 \sqrt{x_{j}^{2}+1}\right]\left[-\sqrt{2}+\frac{2 x_{j}}{\sqrt{x_{j}^{2}+1}}\right] \mathrm{d} t .
$$




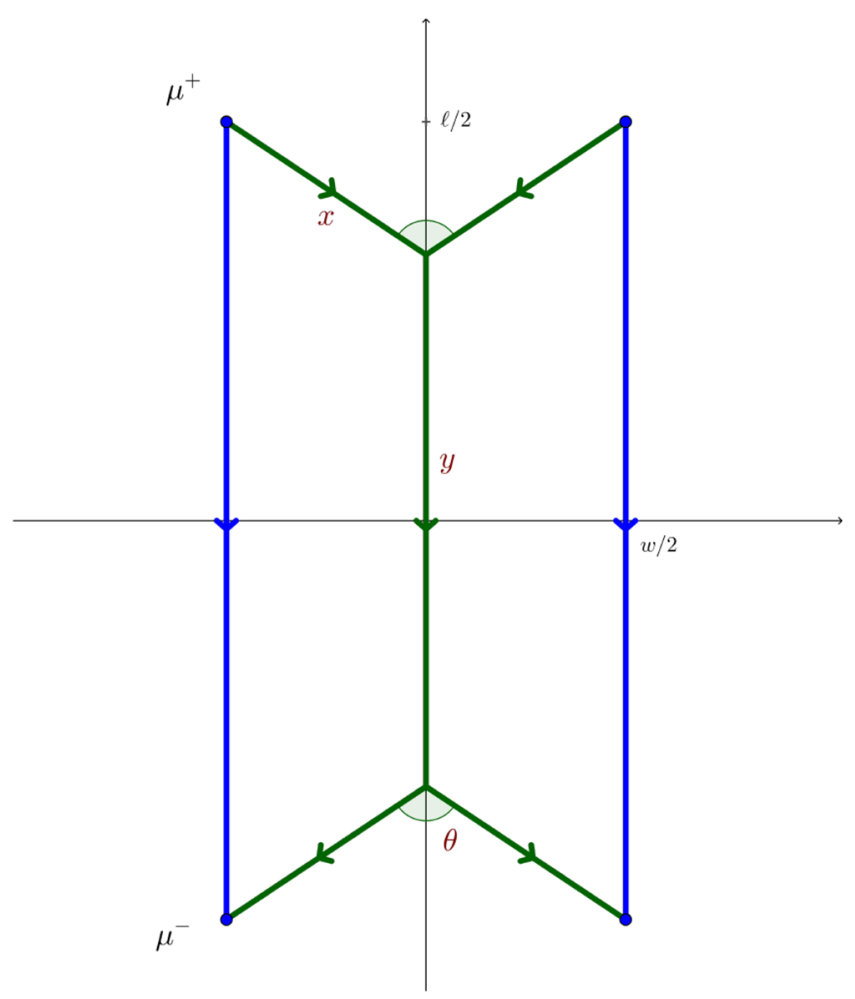

Figure 10. Both $P_{s}$ and $P_{b}$ are depicted here.

Hence, the rate of zipping is given by $x(t)$ which solves the following first order, non-linear ordinary differential equation: $x(0)=2$ and

$$
\frac{\mathrm{d} x}{\mathrm{~d} t}=-\left[\sqrt{2}\left(2-x_{j}\right)+2 \sqrt{x_{j}^{2}+1}\right]\left[-\sqrt{2}+\frac{2 x_{j}}{\sqrt{x_{j}^{2}+1}}\right] .
$$

\subsubsection{Two sources; two sinks}

Suppose that $\mu^{+}=m \delta_{(-w / 2, \ell / 2)}+m \delta_{(w / 2, \ell / 2)}$ and $\mu^{-}=m \delta_{(-w / 2,-\ell / 2)}+m \delta_{(w / 2,-\ell / 2)}$, for $m, w, \ell \in \mathbb{R}^{+}$. That is, the sources and sinks lie on the vertices of a rectangle of width $w$ and length $\ell$. There are two possible optimal transportation networks for $\alpha \in[0,1]$ depending on the configuration of $\mu^{+}$and $\mu^{-}$: a branching path (denoted $\left.P_{b}\right)$ or one composed of two straight edges (denoted $\left.P_{s}\right)$. Both are drawn in Figure 10. For $\alpha \in(0,1]$, we will now consider the flow acting on two different initial paths from $\mu^{+}$to $\mu^{-}$: one with an "X" shape and the other with a "| " shape. The initial network having "| " shape demonstrates that our minimizing flow merely finds local optimal solutions similar to other minimizing or gradient descent flows. However, we will see that the "X" shaped initial network always flows to the global optimal solution regardless of the configuration of the boundary.

Example 4.9. Suppose $T_{0}$ is a transport path directly connecting each source to a sink in a "I " shaped path. This network fails to evolve under the flow regardless of the value of $\ell$. That is to say, for every $h>0$, each $T_{j}^{h}$ must be two parallel, directed line segments; and thus, $T_{t}$ for almost every $t>0$ will stay two parallel directed line segments. (See Fig. 11 for a representation of the two dimensional flow $S$ whose slices are these $T_{t}$.) Hence, the flow finds a local minimum in this case. 


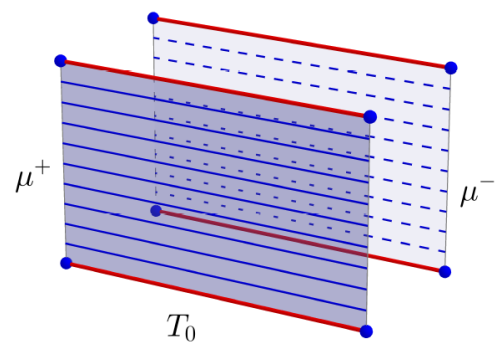

FIGURE 11. Here is a representation of the constructed two dimensional flow $S$ for Example 4.9. We cut it off at some finite time.

One may see this by examining the functional in equation (3.1) in Section 3. With any perturbation of the previous path $T_{j-1}^{h}$, one would increase length of the directed segments and thus, the $\mathbf{M}^{\alpha}$ mass of the path, while simultaneously forcing a positive $\mathbf{F i l l}^{\alpha}$ term. Hence, for every fixed $h>0$, the discrete sequence must only be comprised of $T_{0}$.

Example 4.10. Although the above initial network merely flowed to local optimal network, an initial path with an "X" shape flows to the global optimal solution regardless of the boundary configuration. We will show how the flow on this initial network always includes the necessary topology change to flow to the global minimum in two specific boundary configurations.

Suppose we instead begin with an "X" shaped transport path $T_{0}$ from $\mu^{+}$to $\mu^{-}$.

If $\ell=w$, then we may choose the minimizer for every $h>0$ and $j>0$ having a " $><$ " shape as in Figure $12 \mathrm{~b}$ to be $T_{j}^{h}$, and hence, construct the flow so that every $T_{t}$ has this same shape for almost every $t>0$. The path stabilizes under the flow once it reaches $P_{s}$, which is the optimal network. In this case, the flow changes the topology of the network as needed to find the global minimum since we lose an interior vertex in the breaking process. (See Fig. 13 for a representation of the two dimensional flow $S$ whose slices are these $T_{t}$.)

If $\ell=4 w$, then we may choose the minimizer for every $h>0$ and $j>0$ having a branching structure as in Figure $12 \mathrm{a}$ to be $T_{j}^{h}$, and hence, construct the flow so that every $T_{t}$ has this same branching structure for almost every $t>0$. In this case, the path simultaneously zips up from both ends until reaching the angles of the optimal network $P_{b}$. Since we gain an interior vertex through the zipping process, the flow again changes the topology of the initial network as needed to find the global minimum. (See Fig. 14 for a representation of the two dimensional flow $S$ whose slices are these $T_{t}$.)

Proof. Using similar convexity arguments as those used in Example 4.5, we may show that the paths must zip up along either axis due to the symmetry of $\mu^{+}$and $\mu^{-}$. Zipping along the $y$-axis would result in an edge with associated weight $2 m$, while zipping along the $x$-axis may be thought of as resulting in an edge with associated weight 0. See Figure 12.

Now, fix $h>0$ and set $T_{0}^{h}=T_{0}$. Denote by $T_{x}$ and $T_{y}$ zipping up an $\epsilon>0$ amount along the $x$-axis and $y$-axis, respectively. Then,

$$
G\left(T_{x}\right)=\left[2 m^{\alpha} \sqrt{\ell^{2}+(w-\epsilon)^{2}}\right]^{2}+\frac{\left[m^{\alpha} \ell \epsilon\right]^{2}}{4 h},
$$

and

$$
G\left(T_{y}\right)=\left[(2 m)^{\alpha} \epsilon+2 m^{\alpha} \sqrt{(\ell-\epsilon)^{2}+w^{2}}\right]^{2}+\frac{\left[m^{\alpha} w \epsilon\right]^{2}}{4 h} .
$$




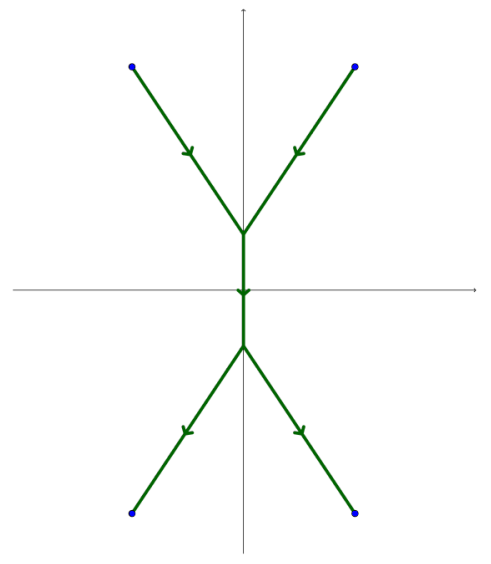

(A) The branching structure when zipping up the $y$-axis.

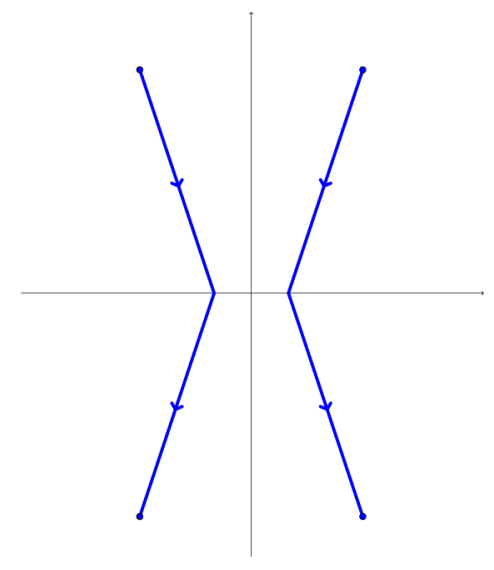

(B) The "><" structure when zipping up the $x$-axis.

FiguRE 12. Comparing $T_{t}$ shapes flowing from an initial "X" shaped network.
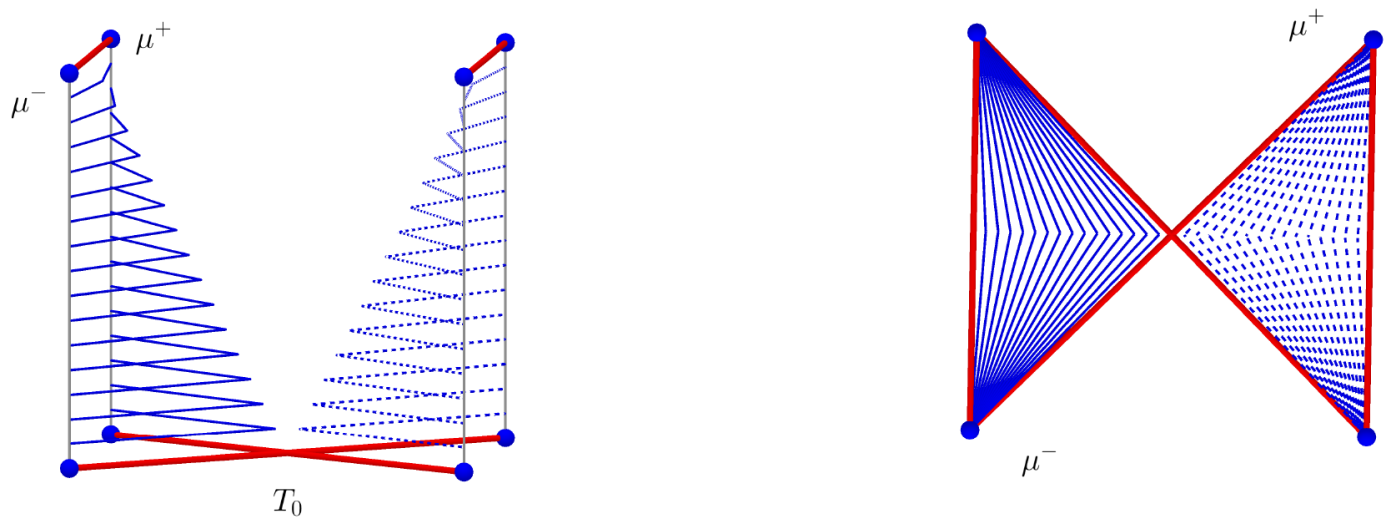

Figure 13. Here is a representation of the constructed, two dimensional flow $S$ when $\ell=w$. (The rate of zipping will affect the shape.) We cut it off at the time when it achieves the optimal shape $P_{s}$.

Let's see how $T_{x}$ reduces cost in the $\ell=w$ case and $T_{y}$ reduces cost in the $\ell=4 w$ case:

If $\ell=w$, then $G_{1}\left(T_{x}\right)<G_{1}\left(T_{y}\right)$ for any $\epsilon>0$. Hence, the initial path breaks apart and flows to $P_{s}$, as desired. See Figure 13.

On the other hand, if $\ell=4 w$, then $G_{1}\left(T_{x}\right)>G_{1}\left(T_{y}\right)$ for sufficiently small $\epsilon>0$. Thus, the initial path must zip up along the $y$-axis and flow to $P_{b}$. See Figure 14 .

Remark 4.11. While we only considered cases when zipping occurs, one may show similar results for paths that must unzip instead. Our constructed flow's ability to alter the topology of the transport network in cost saving ways is due to our choice of functional $G$ defined in equation (3.1) determining the chains in the step minimizing sequences. 


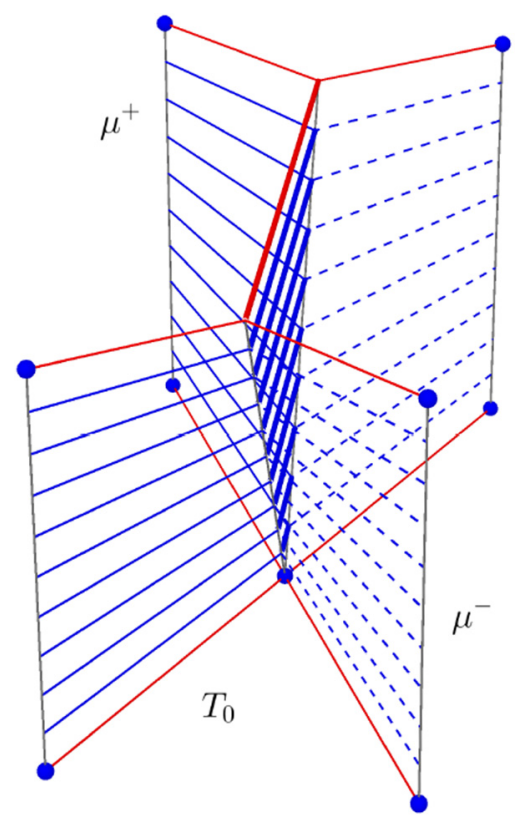

Figure 14. Here is a representation of the constructed, two dimensional flow $S$ when $\ell=4 w$. (The rate of zipping will affect the shape.) We cut it off at the time when it achieves the optimal shape $P_{b}$.

\section{OPEN QUESTIONS}

Constructing optimal paths between atomic measures via current techniques proves computationally difficult often due to the vast number of possible topologies. Since this $\mathbf{M}^{\alpha}$ mass reducing flow is able to change the topology of the initial network, one hopes that the flow will overcome this difficulty, solving the Gilbert transport optimization problem. Unfortunately, Example 4.9 shows that the flow generally finds merely local minima. However, Example 4.10 leads us to conjecture that we may hope to pick a portfolio of initial paths with at least one flowing to an optimal transportation network. We hope that the topology will evolve as needed to move towards the global optimal transport network by "breaking apart" and zipping up when starting with similar "coned" initial networks as that in Example 4.10.

Conjecture 5.1. For atomic measures $\mu^{+}$and $\mu^{-}$, an initial starting transport path composed of "coned" networks (having "hubs" for every anticipated connected component of an optimal path) will flow to an optimal transport network from $\mu^{+}$to $\mu^{-}$.

Because of the results shown in the above examples, we additionally hope that existing discrete computational methods may be enhanced by incorporating the ideas in functional $G$ in such ways that allow for detection of topological modifications for cost reduction.

\section{REFERENCES}

[1] F. Almgren, Deformations and multiple-valued functions, Geometric measure theory and the calculus of variations. Proc. Sympos. Pure Math, American Math. Soc. 44 (1986) 29-130.

[2] F. Almgren, Plateau's problem. Mathematics monograph series. W. A. Benjamin, Inc. (1966).

[3] F. Almgren and J. Taylor, Flat flow is motion by crystalline curvature for curves with crystalline energies. J. Differ. Geometry 42 (1995) 1-22.

[4] F. Almgren, J. Taylor and L. Wang, Curvature-driven flows: a variational approach. SIAM J. Control Optim. 31 (1993) $387-483$. 
[5] M. Bernot, V. Caselles and J.-M. Morel, Optimal transportation networks. Lecture Notes in Mathematics 1955. Springer (2009).

[6] M. Bernot, Irrigation and optimal transport. Ph.D. Thesis, Ecole Normale Superieure de Cachan (2005).

[7] K.A. Brakke, The motion of a surface by its mean curvature. Princeton University Press (1978).

[8] X. Cheng, A mass reducing flow for integral currents, Indiana Univ. Math. J. 42 (1993) 425-444.

[9] O. Chodosh, Brian White - Topics in GMT (MATH 258) Lecture Notes, Stanford University. Available from: http://web.stanford.edu/ ochodosh/GMTnotes.pdf (Spring 2012).

[10] T. Colding, W. Minicozzi and E. Pedersen, Mean curvature flow. Bull. Am. Math. Soc. 52 (2015) $297-333$.

[11] J. Douglas, Minimal surfaces of higher topological structure. Ann. Math. (1939) 205-298.

[12] T. De Pauw and R. Hardt, Rectifiable and flat g chains in a metric space. Am. J. Math. 134 (2012) 1-69.

[13] L. Evans and J. Spruck, Motion of level sets by mean curvature I. J. Differ. Geometry 33 (1991) 635-681.

[14] L. Evans and J. Spruck, Motion of level sets by mean curvature II. Trans. Am. Math. Soc. 330 (1992) 32-1-332.

[15] L. Evans and J. Spruck, Motion of level sets by mean curvature III. J. Geometric Anal. 2 (1992) 121-150.

[16] L. Evans and J. Spruck, Motion of level sets by mean curvature IV. J. Geometric Anal. 5 (1995) 77-114.

[17] H. Federer, Geometric measure theory. Springer-Verlag (1969).

[18] H. Federer and W.H. Fleming, Normal and integral currents. Ann. Math. (1960) 458-520.

[19] H. Federer, Real flat chains, cochains and variational problems. Indiana Univ. Math. J. 24 (1974) 351-407.

[20] W.H. Fleming, Flat chains over a finite coefficient group. Trans. Am. Math. Soc. 121 (1966) 160-186.

[21] M. Gage and R. Hamilton, The heat equation shrinking convex plane curves. J. Differ. Geometry 23 (1986) 69-96.

$[22]$ E.N. Gilbert, Minimum cost communication networks. Bell Labs Tech. J. 46 (1967) 2209-2227.

[23] M. Grayson, The heat equation shrinks embedded plane curves to round points. J. Differ. Geometry 26 (1987) $285-314$.

[24] J. Haga, K. Hoshino and N. Kikuchi, Construction of harmonic map flows through the method of discrete Morse flows. Comput. Visual. Sci. 7 (2004) 53-59.

[25] G. Huisken, Flow by mean curvature of convex surfaces into spheres. J. Differ. Geometry 20 (1984) $237-266$.

[26] L.V. Kantorovich, On the translocation of masses. Dokl. Akad. Nauk SSSR 37 (1942) 199-201.

[27] F. Maddalena, J.-M. Morel and S. Solimini, A variational model of irrigation patterns. Interfaces Free Boundaries 5 (2003) 391-415.

[28] G. Monge, Mémoire sur la théorie des déblais et de remblais. Histoire de l'Académie Royale des Sciences de Paris (1781) 666-704.

[29] F. Morgan, Geometric measure theory. Academic Press (2009).

[30] E. Oudet and F. Santambrogio, A Modica-Mortola approximation for brached transport and applications. Arch. Ratl. Mech. Anal. 201 (2011) 115-142.

[31] P. Pegon, F. Santambrogio and Q. Xia, A fractal shape optimization problem in branched transport. J. Math. Pures Appl. (2017).

[32] J.A.F. Plateau, Statique expérimentale et théoreique des liquides soumis aux seules forces molécaires 2, Gauthier-Villars (1873).

[33] T. Radó, The problem of the least area and the problem of Plateau. Math. Zeitschrift 32 (1930) $763-796$.

[34] E.R. Reifenberg, Solution of the Plateau problem form-dimensional surfaces of varying topological type. Acta Math. 104 (1960) $1-92$.

[35] F. Santambrogio, Optimal channel networks, landscape function and branched transport. Interfaces Free Bound 9 (2007) 149-169.

[36] H. Whitney, Geometric integration theory. Princeton University Press (1957).

[37] B. White, The deformation theorem for flat chains. Acta Math. 183 (1999) 255-271.

[38] B. White, Rectifiability of flat chains. Ann. Math. 150 (1999) 165-184.

[39] B. White, Evolution of curves and surfaces by mean curvature. Proc. ICM (2002).

[40] B. White, Currents and flat chains associated to varifolds, with an application to mean curvature flow. Duke Math J. 148 (2009) 41-62.

[41] Q. Xia, Optimal paths related to transport problems. Commun. Contemp. Math. 5 (2003) 251-279.

[42] Q. Xia, Interior regularity of optimal transport paths. Calc. Variat. Partial Differ. Equ. 20 (2004) $283-299$.

[43] Q. Xia, An application of optimal transport paths to urban transport networks. Discrete Continu. Dyn. Syst. (2005) 904-910.

[44] Q. Xia, The formation of a tree leaf. ESAIM: COCV 13 (2007) 359-377.

[45] Q. Xia, Boundary regularity of optimal transport paths. Adv. Calc. Variat. 4 (2011) 153-174.

[46] Q. Xia, Ramified optimal transportation in geodesic metric spaces. Adv. Calc. Variat. 4 (2011) $277-307$.

[47] Q. Xia, On landscape functions associated with transport paths. Discrete Continu. Dyn. Syst. 34 (2014) $1683-1700$.

[48] Q. Xia, Motivations, ideas, and applications of ramified optimal transportation. ESAIM: M2AN 49 (2015) $1791-1832$.

[49] Q. Xia and A. Vershynina, On the transport dimension of measures. SIAM J. Math. Anal. 41 (2010) $2407-2430$.

[50] Q. Xia and S. Xu, The exchange value embedded in a transport system. Appl. Math. Optim. 62 (2010) $229-252$. 